Israel J. Math. (to appear)

\title{
Characterizations of BMO Associated with Gauss Measures via Commutators of Local Fractional Integrals
}

\author{
Liguang Liu and Dachun Yang *
}

\begin{abstract}
Let $d \gamma(x) \equiv \pi^{-n / 2} e^{-|x|^{2}} d x$ for all $x \in \mathbb{R}^{n}$ be the Gauss measure on $\mathbb{R}^{n}$. In this paper, the authors establish the characterizations of the space $\operatorname{BMO}(\gamma)$ of Mauceri and Meda via commutators of either local fractional integral operators or local fractional maximal operators. To this end, the authors first prove that such a local fractional integral operator of order $\beta$ is bounded from $L^{p}(\gamma)$ to $L^{p /(1-p \beta)}(\gamma)$, or from the Hardy space $H^{1}(\gamma)$ of Mauceri and Meda to $L^{1 /(1-\beta)}(\gamma)$ or from $L^{1 / \beta}(\gamma)$ to $\operatorname{BMO}(\gamma)$, where $\beta \in(0,1)$ and $p \in(1,1 / \beta)$.
\end{abstract}

\section{Introduction}

The space $\mathrm{BMO}\left(\mathbb{R}^{n}\right)$ of functions with bounded mean oscillation was first introduced by John and Nirenberg [13] and it plays an important role in harmonic analysis and partial differential equations; see, for example, $[23,8,21]$. One of the remarkable characterizations of the space $\mathrm{BMO}\left(\mathbb{R}^{n}\right)$ is given in terms of commutators of certain operators. In particular, when $T$ is a singular integral with standard kernel, Coifman, Rochberg and Weiss [4] proved that $b \in \operatorname{BMO}\left(\mathbb{R}^{n}\right)$ is sufficient for $[b, T](f) \equiv b T(f)-T(b f)$ to be bounded on $L^{p}\left(\mathbb{R}^{n}\right)$ with $p \in(1, \infty)$ and also established a partial converse. The full converse of this result was obtained by Janson [12]. Moreover, assuming that $I_{\beta}$ is a fractional integral operator of order $\beta$ with $\beta \in(0, n)$ (see, for example, [20, p. 116]), Chanillo [3] proved that $\left[b, I_{\beta}\right]$ is bounded from $L^{p}\left(\mathbb{R}^{n}\right)$ to $L^{q}\left(\mathbb{R}^{n}\right)$ if and only if $b \in \mathrm{BMO}\left(\mathbb{R}^{n}\right)$, where $1<p<q<\infty$ and $1 / q=1 / p-\beta / n$.

The main purpose of this paper is to generalize the above result of Chanillo [3] to the setting of the Gauss measure metric space $\left(\mathbb{R}^{n},|\cdot|, d \gamma\right)$, where $|\cdot|$ denotes the Euclidean norm and $d \gamma(x) \equiv \pi^{-n / 2} e^{-|x|^{2}} d x$ for all $x \in \mathbb{R}^{n}$ the Gauss measure. Such an underlying space $\left(\mathbb{R}^{n},|\cdot|, d \gamma\right)$ naturally appears in the analysis associated with the Ornstein-Uhlenbeck operator; see $[18,9,19,7,17,16]$ and the references therein. However, $\left(\mathbb{R}^{n},|\cdot|, d \gamma\right)$ is not a space of homogeneous type in the sense of Coifman and Weiss [5, 6]. Recently, Mauceri and Meda [16] developed a theory of singular integrals on $\left(\mathbb{R}^{n},|\cdot|, d \gamma\right)$ which plays for the Ornstein-Uhlenbeck operator the same role as that the theory of classical CalderónZygmund operators plays for the Laplacian on classical Euclidean spaces. The approach

2000 Mathematics Subject Classification. Primary 47B47; Secondary 47H50, $42 B 30$.

Key words and phrases. Gauss measure, $\mathrm{BMO}(\gamma)$, fractional integral operator, fractional maximal operator, commutator.

The second author is supported by the National Natural Science Foundation (Grant No. 10871025) of China.

${ }^{*}$ Corresponding author. 
used in [16] requires the introduction of certain Hardy space $H^{1}(\gamma)$ and its dual space $\operatorname{BMO}(\gamma)$ related to a certain class $\mathcal{B}_{a}$ with $a \in(0, \infty)$ of admissible balls.

In this paper, we characterize the space $\operatorname{BMO}(\gamma)$ of Mauceri and Meda [16] via commutators of either local fractional integral operators or local fractional maximal operators. To this end, we first establish the boundedness of such a local fractional integral operator on Lebesgue spaces or the corresponding Hardy space and its dual space. A main difficulty to obtain these results exists in the non-doubling property of the Gauss measure.

To state our results, we first recall some notation and notions; see, for example, [16]. Let $m(x) \equiv \min \{1,1 /|x|\}$ for all $x \in \mathbb{R}^{n}$. We denote by $c_{B}$ and $r_{B}$, respectively, the center and radius of any ball $B$. For any $\kappa>0$, denote by $\kappa B$ the ball with center $c_{B}$ and radius $\kappa r_{B}$. Let $a \in(0, \infty)$. The admissible class $\mathcal{B}_{a}$ of balls is defined to be the set of all balls $B \subset \mathbb{R}^{n}$ such that $r_{B} \leq a m\left(c_{B}\right)$. For any $a \in(0, \infty)$ and $x \in \mathbb{R}^{n}$, denote by $\mathcal{B}_{a}(x)$ the collection of all balls $B \in \mathcal{B}_{a}$ containing $x$.

Mauceri and Meda [16, p. 281] introduced the following $\operatorname{BMO}(\gamma)$ space. Precisely, a function $f \in L^{1}(\gamma)$ is said to belong to the $\operatorname{space} \operatorname{BMO}(\gamma)$ if

$$
\|f\|_{*} \equiv \sup _{B \in \mathcal{B}_{1}} \frac{1}{\gamma(B)} \int_{B}\left|f(x)-f_{B}\right| d \gamma(x)<\infty
$$

where and in what follows, $\gamma(B)$ denotes the Gauss measure of $B$ and

$$
f_{B} \equiv \frac{1}{\gamma(B)} \int_{B} f(y) d \gamma(y)
$$

Moreover, the $\operatorname{BMO}(\gamma)$ norm of $f$ is defined by

$$
\|f\|_{\mathrm{BMO}(\gamma)} \equiv\|f\|_{*}+\|f\|_{L^{1}(\gamma)} .
$$

Mauceri and Meda [16] also introduced the atomic Hardy space $H^{1}(\gamma)$, which is the predual space of $\operatorname{BMO}(\gamma)$; see [16, Theorem 5.2]. Precisely, assume $r \in(1, \infty)$. A $(1, r)$ atom is either the constant function 1 , or a function $a \in L^{1}(\gamma)$ supported in a ball $B \in \mathcal{B}_{1}$ with the properties $\|a\|_{L^{r}(\gamma)} \leq[\gamma(B)]^{1 / r-1}$ and $\int_{B} a(x) d \gamma(x)=0$. The Hardy space $H^{1, r}(\gamma)$ is the space of all functions $g \in L^{1}(\gamma)$ that admits a decomposition of the form

$$
g=\sum_{k=1}^{\infty} \lambda_{k} a_{k}
$$

where $\left\{a_{k}\right\}_{k=1}^{\infty}$ are $(1, r)$ atoms and $\left\{\lambda_{k}\right\}_{k=1}^{\infty} \subset \mathbb{C}$ satisfying that $\sum_{k=1}^{\infty}\left|\lambda_{k}\right|<\infty$. The norm $\|g\|_{H^{1, r}(\gamma)}$ of $g$ is defined to be the infimum of $\sum_{k=1}^{\infty}\left|\lambda_{k}\right|$ over all decompositions of $g$ as in (1.2). It was pointed out in [16, p. 297] that the Hardy spaces $H^{1, r}(\gamma)$ for all $r \in(1, \infty)$ coincide with equivalent norms, which will be simply denoted by $H^{1}(\gamma)$.

Motivated by geometry properties of the Gauss measure, especially the fact that the Gauss measure is doubling on each class of admissible balls $\mathcal{B}_{a}$ (see [16, Proposition 2.1]), for any given $a \in(0, \infty)$ and $\beta \in(0,1)$, we define the local fractional integral operator $I_{a}^{\beta}$ by that for all functions $f \in L_{c}^{\infty}(\gamma)$ and $x \in \mathbb{R}^{n}$,

$$
I_{a}^{\beta}(f)(x) \equiv \int_{B(x, a m(x))} \frac{f(y)}{[V(x, y)]^{1-\beta}} d \gamma(y),
$$


where and in what follows, $V(x, y) \equiv \gamma(B(x,|x-y|))$ and $L_{c}^{\infty}(\gamma)$ denotes the set of all functions in $L^{\infty}(\gamma)$ with compact support. In fact, obviously, $L^{\infty}(\gamma)=L^{\infty}\left(\mathbb{R}^{n}\right)$ and $L_{c}^{\infty}(\gamma)=L_{c}^{\infty}\left(\mathbb{R}^{n}\right)$ with equivalent norms. To characterize the space $\operatorname{BMO}(\gamma)$, we also introduce a variant of the above local fractional integral operator $I_{a}^{\beta}$, which we denote by $\widetilde{I}_{a}^{\beta}$. Precisely, for all functions $f \in L_{c}^{\infty}(\gamma)$ and $x \in \mathbb{R}^{n}$,

$$
\widetilde{I}_{a}^{\beta}(f)(x) \equiv \int_{B(x, a m(x))} \frac{f(y)}{\left[e^{\left.-|x|^{2}|x-y|^{n}\right]^{1-\beta}}\right.} d \gamma(y) .
$$

By (2.3) below, it is not difficult to see that there exists a positive constant $C$, depending only on $n$ and $a$, such that for all $x \in \mathbb{R}^{n}$ and $y \in B(x, a m(x))$,

$$
C^{-1} V(x, y) \leq e^{-|x|^{2}}|x-y|^{n} \leq C V(x, y) .
$$

Hence, when $f$ is a non-negative function, $I_{a}^{\beta}(f)$ and $\widetilde{I}_{a}^{\beta}(f)$ are pointwisely equivalent.

It is proved in Theorems 3.1 and 3.2 below that both $I_{a}^{\beta}$ and $\widetilde{I}_{a}^{\beta}$ are bounded from $L^{p}(\gamma)$ to $L^{p /(1-p \beta)}(\gamma)$ when $p \in(1,1 / \beta)$, or from $H^{1}(\gamma)$ to $L^{1 /(1-\beta)}(\gamma)$, or from $L^{1 / \beta}(\gamma)$ to $\operatorname{BMO}(\gamma)$ (actually, from $\left\{f \in L^{1 / \beta}(\gamma): f \geq 0\right\}$ to $\operatorname{BLO}_{a}(\gamma)$, where $\mathrm{BLO}_{a}(\gamma)$ was introduced in [14] and $\left.\mathrm{BLO}_{a}(\gamma) \subsetneq \mathrm{BMO}(\gamma)\right)$. These results of boundedness are of independent interest; see $[1,22]$ for the corresponding boundedness results of the classical fractional integral operators.

If $b \in \operatorname{BMO}(\gamma)$, then the commutator $\left[b, \widetilde{I}_{a}^{\beta}\right]$, generated by $b$ and the local fractional integral operator $\widetilde{I}_{a}^{\beta}$, is defined by setting, for all functions $f \in L_{c}^{\infty}(\gamma)$,

$$
\left[b, \widetilde{I}_{a}^{\beta}\right](f) \equiv b \widetilde{I}_{a}^{\beta}(f)-\widetilde{I}_{a}^{\beta}(b f) .
$$

Moreover, we define $\widetilde{\left[b, I_{a}^{\beta}\right]}$ by setting, for all functions $f \in L_{c}^{\infty}(\gamma)$ and all $x \in \mathbb{R}^{n}$,

$$
\widetilde{\left[b, I_{a}^{\beta}\right]}(f)(x) \equiv \int_{B(x, a m(x))} \frac{|b(x)-b(y)||f(y)|}{[V(x, y)]^{1-\beta}} d \gamma(y) .
$$

Applying the boundedness of $I_{a}^{\beta}$ and $\widetilde{I}_{a}^{\beta}$ from $L^{p}(\gamma)$ to $L^{p /(1-p \beta)}(\gamma)$ with $p \in(1,1 / \beta)$, we characterize the space $\operatorname{BMO}(\gamma)$ by these commutators as follows.

Theorem 1.1 Let $a \in(0, \infty), \beta \in(0,1), 1<p<q<\infty$ and $1 / q=1 / p-\beta$. Then there exists a positive constant $C$, depending on $a, p$ and $q$, such that the following hold.

(i) If $b \in \operatorname{BMO}(\gamma)$, then for all $f \in L_{c}^{\infty}(\gamma)$,

$$
\left\|\widetilde{\left[b, I_{a}^{\beta}\right]}(f)\right\|_{L^{q}(\gamma)} \leq C\|b\|_{*}\|f\|_{L^{p}(\gamma)} .
$$

Moreover, the sublinear operator $\widetilde{\left[b, I_{a}^{\beta}\right]}$ admits a unique bounded extension from $L^{p}(\gamma)$ to $L^{q}(\gamma)$ with norm at most a constant multiple of $\|b\|_{*}$.

(ii) If $b \in L^{1}(\gamma)$ and $\left[b, \widetilde{I}_{a}^{\beta}\right]$ is bounded from $L^{p}(\gamma) \cap L_{c}^{\infty}(\gamma)$ to $L^{q}(\gamma)$, then $b \in \operatorname{BMO}(\gamma)$ and

$$
\|b\|_{\operatorname{BMO}(\gamma)} \leq\|b\|_{L^{1}(\gamma)}+C\left\|\left[b, \widetilde{I}_{a}^{\beta}\right]\right\|_{L^{p}(\gamma) \rightarrow L^{q}(\gamma)} .
$$


The proof of Theorem 1.1 is given in Section 4. Observe that there exists a positive constant $C$ such that for all $x \in \mathbb{R}^{n}$,

$$
\left|\left[b, \widetilde{I}_{a}^{\beta}\right](f)(x)\right| \leq \widetilde{C\left[I_{1}^{\beta}\right]}(f)(x) .
$$

Thus, Theorem 1.1 actually implies that the boundedness of either $\left[b, \widetilde{I}_{a}^{\beta}\right]$ or $\widetilde{\left[b, I_{a}^{\beta}\right]}$ characterizes $b \in \operatorname{BMO}(\gamma)$. Moreover, for $a \in(0, \infty)$ and $\beta \in(0,1)$, if we define the dual operator $\left(I_{a}^{\beta}\right)^{*}$ of $I_{a}^{\beta}$ by setting, for all functions $f \in L_{c}^{\infty}(\gamma)$ and $x \in \mathbb{R}^{n}$,

$$
\left(I_{a}^{\beta}\right)^{*}(f)(x) \equiv \int_{B(y, a m(y))} \frac{f(y)}{[V(y, x)]^{1-\beta}} d \gamma(y)
$$

and define the dual operator $\left(\widetilde{I}_{a}^{\beta}\right)^{*}$ of $\widetilde{I}_{a}^{\beta}$ by setting, for all functions $f \in L_{c}^{\infty}(\gamma)$ and $x \in \mathbb{R}^{n}$,

$$
\left(\widetilde{I}_{a}^{\beta}\right)^{*}(f)(x) \equiv \int_{B(y, a m(y))} \frac{f(y)}{\left[e^{\left.-|y|^{2}|x-y|^{n}\right]^{1-\beta}}\right.} d \gamma(y),
$$

then Theorem 1.1 and all results in Section 3 related to $I_{a}^{\beta}$ and $\widetilde{I}_{a}^{\beta}$ are also true for $\left(I_{a}^{\beta}\right)^{*}$ and $\left(\widetilde{I}_{a}^{\beta}\right)^{*}$; we omit the details by similarity.

For any given $a \in(0, \infty)$ and $\beta \in(0,1)$, we define the local fractional maximal operator $\mathcal{M}_{a}^{\beta}$ by setting, for all locally integrable functions $f$ and all $x \in \mathbb{R}^{n}$,

$$
\mathcal{M}_{a}^{\beta}(f)(x) \equiv \sup _{B \in \mathcal{B}_{a}(x)} \frac{1}{[\gamma(B)]^{1-\beta}} \int_{B}|f(y)| d \gamma(y) .
$$

The boundedness results for $\mathcal{M}_{a}^{\beta}$ are presented in Corollary 3.1 below.

If $b \in \operatorname{BMO}(\gamma)$, then the commutator $\left[b, \mathcal{M}_{a}^{\beta}\right]$, generated by $b$ and the local fractional maximal operator $\mathcal{M}_{a}^{\beta}$, is defined by setting, for all functions $f \in L_{c}^{\infty}(\gamma)$,

$$
\left[b, \mathcal{M}_{a}^{\beta}\right](f) \equiv b \mathcal{M}_{a}^{\beta}(f)-\mathcal{M}_{a}^{\beta}(b f) .
$$

Correspondingly, we define $\left[\widetilde{b, \mathcal{M}_{a}^{\beta}}\right]$ by setting, for all functions $f \in L_{c}^{\infty}(\gamma)$ and all $x \in \mathbb{R}^{n}$,

$$
\left.\widetilde{\left[b, \mathcal{M}_{a}^{\beta}\right.}\right](f)(x) \equiv \sup _{B \in \mathcal{B}_{a}(x)} \frac{1}{[\gamma(B)]^{1-\beta}} \int_{B}|b(x)-b(y)||f(y)| d \gamma(y)
$$

Applying Theorem 1.1, we also obtain the following characterization of the space $\operatorname{BMO}(\gamma)$

Theorem 1.2 Let $a \in(0, \infty), \beta \in(0,1), 1<p<q<\infty$ and $1 / q=1 / p-\beta$. Then there exists a positive constant $C$, depending on $a, p$ and $q$, such that the following hold.

(i) If $b \in \operatorname{BMO}(\gamma)$, then for all $f \in L_{c}^{\infty}(\gamma)$,

$$
\left.\| \widetilde{\left[b, \mathcal{M}_{a}^{\beta}\right.}\right](f)\left\|_{L^{q}(\gamma)} \leq C\right\| b\left\|_{*}\right\| f \|_{L^{p}(\gamma)} .
$$

Moreover, the sublinear operator $\left[\widetilde{\left[\mathcal{M}_{a}^{\beta}\right.}\right]$ admits a unique bounded extension from $L^{p}(\gamma)$ to $L^{q}(\gamma)$ with norm at most a constant multiple of $\|b\|_{*}$. 
(ii) If $b \in L^{1}(\gamma)$ and $\left.\widetilde{\left[b, \mathcal{M}_{a}^{\beta}\right.}\right]$ is bounded from $L^{p}(\gamma) \cap L_{c}^{\infty}(\gamma)$ to $L^{q}(\gamma)$, then $b \in \operatorname{BMO}(\gamma)$ and

$$
\|b\|_{\mathrm{BMO}(\gamma)} \leq\|b\|_{L^{1}(\gamma)}+C\left\|\widetilde{\left[b, \mathcal{M}_{a}^{\beta}\right]}\right\|_{L^{p}(\gamma) \rightarrow L^{q}(\gamma)} .
$$

For any given function $f$ and all $x \in \mathbb{R}^{n}$, we set $f^{+}(x) \equiv \max \{f(x), 0\}$ and $f^{-}(x) \equiv$ $-\min \{0, f(x)\}$. Motivated by [2], we obtain the following result.

Theorem 1.3 Let $a \in(0, \infty), \beta \in(0,1), 1<p<q<\infty$ and $1 / q=1 / p-\beta$. Then there exists a positive constant $C$, depending on $a, p$ and $q$, such that the following hold.

(i) If $b \in \operatorname{BMO}(\gamma)$ and $b^{-} \in L^{\infty}(\gamma)$, then for all $f \in L_{c}^{\infty}(\gamma)$,

$$
\left\|\left[b, \mathcal{M}_{a}^{\beta}\right](f)\right\|_{L^{q}(\gamma)} \leq C\left(\|b\|_{*}+\left\|b^{-}\right\|_{L^{\infty}(\gamma)}\right)\|f\|_{L^{p}(\gamma)} .
$$

(ii) If $b \in L^{1}(\gamma)$ and $\left[b, \mathcal{M}_{a}^{\beta}\right]$ is bounded from $L^{p}(\gamma) \cap L_{c}^{\infty}(\gamma)$ to $L^{q}(\gamma)$, then $b \in \operatorname{BMO}(\gamma)$, $b^{-} \in L^{\infty}(\gamma)$ and

$$
\|b\|_{\mathrm{BMO}(\gamma)}+\left\|b^{-}\right\|_{L^{\infty}(\gamma)} \leq\|b\|_{L^{1}(\gamma)}+C\left\|\left[b, \mathcal{M}_{a}^{\beta}\right]\right\|_{L^{p}(\gamma) \rightarrow L^{q}(\gamma)}
$$

where

$$
\left\|\left[b, \mathcal{M}_{a}^{\beta}\right]\right\|_{L^{p}(\gamma) \rightarrow L^{q}(\gamma)}=\sup \left\{\left\|\left[b, \mathcal{M}_{a}^{\beta}\right](f)\right\|_{L^{q}(\gamma)}: f \in L_{c}^{\infty}(\gamma),\|f\|_{L^{p}(\gamma)}=1\right\} .
$$

We point out that in Theorem $1.3(\mathrm{i})$, since the operator $\left[b, \mathcal{M}_{a}^{\beta}\right]$ as in (1.6) is not even sublinear, we can not naturally extend the domain of $\left[b, \mathcal{M}_{a}^{\beta}\right]$ to the whole $L^{p}(\gamma)$.

Finally, we make the following conventions on notation. Let $\mathbb{N} \equiv\{1,2, \cdots\}$. Define $p^{\prime}$ to be the conjugate value of $p$, namely, $1 / p+1 / p^{\prime}=1$ for $p \in[1, \infty]$. Denote by $\chi_{E}$ the characterize function of any set $E \subset \mathbb{R}^{n}$. We also denote by $C$ a positive constant independent of main parameters involved, which may vary at different occurrences. Constants with subscripts do not change through the whole paper. We use $f \lesssim g$ to denote $f \leq C g$. If $f \lesssim g \lesssim f$, we then write $f \sim g$. For any given "normed" spaces $\mathcal{X}$ and $\mathcal{Y}$, an operator $T$ is said to be bounded from $\mathcal{X}$ to $\mathcal{Y}$ means that there exists a nonnegative constant $C$ such that for all $f \in \mathcal{X}$ and $T(f) \in \mathcal{Y},\|T(f)\|_{\mathcal{Y}} \leq C\|f\|_{\mathcal{X}}$; moreover, we denote by $\|T\|_{\mathcal{X} \rightarrow \mathcal{Y}}$ the operator norm of $T$. For any $b \in L_{\text {loc }}^{1}(\gamma)$ and any ball $B \subset \mathbb{R}^{n}$, set $b_{B} \equiv \frac{1}{\gamma(B)} \int_{B} b(y) d \gamma(y)$.

\section{Preliminaries}

Some geometry properties concerned with the Gauss measure are used throughout the whole paper. An important one, among others, is that the Gauss measure is indeed doubling on all balls in $\mathcal{B}_{a}$. Precisely, for all $\tau, a \in(0, \infty)$ and $B \in \mathcal{B}_{a}$, denote by $B_{\tau}^{*}$ the union of all balls $B^{\prime}$ that intersect $B$ such that $r_{B^{\prime}} \leq \tau r_{B}$. It was proved in $[16$, Proposition 2.1] that

$$
\sigma_{a, \tau}^{*} \equiv \sup _{B \in \mathcal{B}_{a}} \frac{\gamma\left(B_{\tau}^{*}\right)}{\gamma(B)} \leq(2 \tau+1)^{n} e^{4 a(\tau+1)+a^{2}}
$$


which is deduced from the property that for all $B \in \mathcal{B}_{a}$ and $x \in B$,

$$
e^{-2 a-a^{2}} \leq e^{\left|c_{B}\right|^{2}-|x|^{2}} \leq e^{2 a} .
$$

Moreover, it follows from (2.2) that for all $B \in \mathcal{B}_{a}$,

$$
\gamma(B) \sim e^{-\left|c_{B}\right|^{2}}|B|
$$

with constant depending only on $a$ and $n$, where $|B|$ denotes the Lebesgue measure of $B$.

Recall that it was proved in [16, (3.4)] that for all $y \in B$ and $B \in \mathcal{B}_{1}, m(y) \leq 2 m\left(c_{B}\right)$. An argument similar to that also yields that for all $B \in \mathcal{B}_{a}$ and $y \in B$,

$$
(a+1)^{-1} m(y) \leq m\left(c_{B}\right) \leq(a+1) m(y)
$$

see also [14]. Using (2.4) and following a procedure similar to that in [7, Lemma 2.4], we obtain the following geometry covering lemma. Here we omit the details.

Lemma 2.1 Let $\kappa=\frac{1}{20}$ and $\left\{B\left(x_{j}, \kappa m\left(x_{j}\right) / 4\right)\right\}_{j \in \mathbb{N}}$ be a maximal family of disjoint balls contained in $\mathbb{R}^{n}$. Set $B_{j} \equiv B\left(x_{j}, \kappa m\left(x_{j}\right)\right)$. Then $\mathbb{R}^{n}=\cup_{j \in \mathbb{N}} B_{j}$. Moreover, for any given $\tau \in(0, \infty)$, there exists a positive constant $N$ depending only on $n$ and $\tau$ such that for all $x \in \mathbb{R}^{n}, \sum_{j \in \mathbb{N}} \chi_{\left(\tau B_{j}\right)}(x) \leq N$.

For any given $a \in(0, \infty)$ and locally integrable function $f$, Mauceri and Meda [16, p. 286] introduced the noncentered local Hardy-Littlewood maximal function $\mathcal{M}_{a} f$ by setting, for all $x \in \mathbb{R}^{n}$,

$$
\mathcal{M}_{a} f(x) \equiv \sup _{B \in \mathcal{B}_{a}(x)} \frac{1}{\gamma(B)} \int_{B}|f(y)| d \gamma(y)
$$

and they also introduced the local sharp maximal function $f^{\sharp}$ of $f$ by setting, for all $x \in \mathbb{R}^{n}$,

$$
f^{\sharp}(x) \equiv \sup _{B \in \mathcal{B}_{1}(x)} \frac{1}{\gamma(B)} \int_{B}\left|f(y)-f_{B}\right| d \gamma(y) .
$$

For any fixed $a \in(0, \infty)$, it is known that $\mathcal{M}_{a}$ is bounded on $L^{p}(\gamma)$ when $p \in(1, \infty]$ and from $L^{1}(\gamma)$ to $L^{1, \infty}(\gamma)$; see [16, Theorem 3.1]. When $p \in[1, \infty)$, it is also proved in [16, Theorem 3.5] that there exists a positive constant $C$ such that for all $f \in L^{p}(\gamma)$,

$$
\|f\|_{L^{p}(\gamma)} \leq C\left\{\|f\|_{L^{1}(\gamma)}+\left\|f^{\sharp}\right\|_{L^{p}(\gamma)}\right\} .
$$

For any $a \in(0, \infty), p \in[1, \infty)$ and locally integrable function $f$, if we set

$$
\|f\|_{*}^{\mathcal{B}_{a}, p} \equiv \sup _{B \in \mathcal{B}_{a}}\left\{\frac{1}{\gamma(B)} \int_{B}\left|f(x)-f_{B}\right|^{p} d \gamma(x)\right\}^{1 / p}
$$


then by [16, Proposition 2.4] and [16, Section 4], it is easy to see that there exists a positive constant $C$, depending on $a, n$ and $p$, but not on $f$, such that

$$
C^{-1}\|f\|_{*} \leq\|f\|_{*}^{\mathcal{B}_{a}, p} \leq C\|f\|_{*} .
$$

Finally, we conclude this section by recalling the BLO-type spaces in [14]. Let $a \in$ $(0, \infty)$. The space $\mathrm{BLO}_{a}(\gamma)$ is defined to be the set of all locally integrable functions $f$ satisfying

$$
\|f\|_{\mathrm{BLO}_{a}(\gamma)} \equiv\|f\|_{L^{1}(\gamma)}+\sup _{B \in \mathcal{B}_{a}}\left[\frac{1}{\gamma(B)} \int_{B} f(y) d \gamma(y)-\operatorname{essinf}_{x \in B} f(x)\right]<\infty .
$$

For any fixed $a \in(0, \infty)$, it was proved in [14] that $\operatorname{BLO}_{a}(\gamma) \subset \operatorname{BMO}(\gamma)$ and there exists a positive constant $C$ depending only on $a$ and $n$ such that for all $f \in \mathrm{BLO}_{a}(\gamma)$,

$$
\|f\|_{\mathrm{BMO}(\gamma)} \leq C\|f\|_{\mathrm{BLO}_{a}(\gamma)},
$$

and that the inclusion $\mathrm{BLO}_{a}(\gamma) \subset \mathrm{BMO}(\gamma)$ is proper.

\section{Local fractional integral and maximal operators}

In this section, we establish the boundedness of $I_{a}^{\beta}, \widetilde{I}_{a}^{\beta}$ and $\mathcal{M}_{a}^{\beta}$ on various spaces. For the Euclidean case of Theorem 3.1 and a variant of Theorem 3.1 on general metric measure spaces, we refer the reader to [11, Theorem 1] and [10, Theorem 5.3], respectively.

Theorem 3.1 Let $a \in(0, \infty), \beta \in(0,1), 1<p<q<\infty$ and $1 / q=1 / p-\beta$. Then both $I_{a}^{\beta}$ and $\widetilde{I}_{a}^{\beta}$ are bounded from $L^{p}(\gamma)$ to $L^{q}(\gamma)$ and from $L^{1}(\gamma)$ to $L^{1 /(1-\beta), \infty}(\gamma)$.

Proof. Observing that $I_{a}^{\beta}(|f|) \sim \widetilde{I}_{a}^{\beta}(|f|)$, we only need to prove the boundedness results for the operator $I_{a}^{\beta}$. To this end, fix $r \in(0, a)$. For any $x \in \mathbb{R}^{n}$, we write

$$
\left|I_{a}^{\beta}(f)(x)\right| \leq \int_{|x-y|<r m(x)} \frac{|f(y)|}{[V(x, y)]^{1-\beta}} d \gamma(y)+\int_{r m(x) \leq|x-y|<a m(x)} \cdots \equiv \mathrm{Z}_{1}+\mathrm{Z}_{2} .
$$

By (2.2) and (2.3), we obtain

$$
\begin{aligned}
\mathrm{Z}_{1} & \leq \sum_{j=0}^{\infty} \int_{2^{-j-1} r m(x) \leq|x-y|<2^{-j} r m(x)} \frac{|f(y)|}{\left[\gamma\left(B\left(x, 2^{-j-1} r m(x)\right)\right)\right]^{1-\beta}} d \gamma(y) \\
& \leq \sigma_{a, 2}^{*} \sum_{j=0}^{\infty}\left[\gamma\left(B\left(x, 2^{-j-1} r m(x)\right)\right)\right]^{\beta} \frac{1}{\gamma\left(B\left(x, 2^{-j} r m(x)\right)\right)} \int_{B\left(x, 2^{-j} r m(x)\right)}|f(y)| d \gamma(y) \\
& \lesssim e^{-|x|^{2} \beta}[r m(x)]^{\beta n} \mathcal{M}_{a}(f)(x) .
\end{aligned}
$$

By Hölder's inequality, we have

$$
\mathrm{Z}_{2} \leq\|f\|_{L^{p}(\gamma)}\left\{\int_{r m(x) \leq|x-y|<a m(x)}\left(\frac{1}{[\gamma(B(x,|x-y|))]^{1-\beta}}\right)^{p^{\prime}} d \gamma(y)\right\}^{1 / p^{\prime}}
$$




$$
\leq\|f\|_{L^{p}(\gamma)}\left\{\sum_{j=0}^{\left\lfloor\log _{2}\left(\frac{a}{r}\right)\right\rfloor} \int_{2^{j} r m(x) \leq|x-y|<2^{j+1} r m(x)} \frac{1}{\left[\gamma\left(B\left(x, 2^{j} r m(x)\right)\right)\right]^{p^{\prime}(1-\beta)}} d \gamma(y)\right\}^{1 / p^{\prime}}
$$

where $\lfloor a\rfloor$ for any $a \in \mathbb{R}$ denotes the maximal integer no more than $a$. Notice that $B\left(x, 2^{j+1} r m(x)\right) \in \mathcal{B}_{2 a}$ for all $0 \leq j \leq\left\lfloor\log _{2}\left(\frac{a}{r}\right)\right\rfloor$. By this, (2.2), (2.3) and $1 / q=1 / p-\beta$, we further obtain

$$
\begin{aligned}
\mathrm{Z}_{2} & \lesssim\|f\|_{L^{p}(\gamma)}\left\{\sum_{j=0}^{\left\lfloor\log _{2}\left(\frac{a}{r}\right)\right\rfloor} 2^{j n\left(1+\beta p^{\prime}-p^{\prime}\right)}[r m(x)]^{n\left(1+\beta p^{\prime}-p^{\prime}\right)} e^{-|x|^{2}\left(1+\beta p^{\prime}-p^{\prime}\right)}\right\}^{1 / p^{\prime}} \\
& \lesssim\|f\|_{L^{p}(\gamma)}[r m(x)]^{n(\beta-1 / p)} e^{-|x|^{2}(\beta-1 / p)}
\end{aligned}
$$

Notice that

$$
e^{-|x|^{2} \beta}[r m(x)]^{n \beta} \mathcal{M}_{a}(f)(x) \leq\|f\|_{L^{p}(\gamma)}[r m(x)]^{n(\beta-1 / p)} e^{-|x|^{2}(\beta-1 / p)}
$$

if and only if

$$
r \leq \frac{1}{m(x)}\left(\frac{\|f\|_{L^{p}(\gamma)} e^{|x|^{2} / p}}{\mathcal{M}_{a}(f)(x)}\right)^{p / n}
$$

Denote by $A_{1}$ the set $x \in \mathbb{R}^{n}$ such that the right-hand side of (3.1) is smaller than $a$. Set $A_{2} \equiv \mathbb{R}^{n} \backslash A_{1}$. If $x \in A_{1}$, we take $r$ equals to the right-hand side of (3.1), and then obtain

$$
\left|I_{a}^{\beta}(f)(x)\right| \lesssim\|f\|_{L^{p}(\gamma)}^{\beta p}\left[\mathcal{M}_{a}(f)(x)\right]^{1-\beta p} .
$$

If $x \in A_{2}$, we then take $r=a / 2$. In this case, $\|f\|_{L^{p}(\gamma)} e^{|x|^{2} / p} \geq[\operatorname{am}(x)]^{n / p} \mathcal{M}_{a}(f)(x)$ for all $x \in A_{2}$. This combined with the estimates of $\mathrm{Z}_{1}$ and $\mathrm{Z}_{2}$ yields that for all $x \in A_{2}$,

$$
\left|I_{a}^{\beta}(f)(x)\right| \lesssim\|f\|_{L^{p}(\gamma)}^{\beta p}\left[\mathcal{M}_{a}(f)(x)\right]^{1-\beta p} .
$$

Then by (3.2), (3.3), $q(1-p \beta)=p$ and the boundedness of $\mathcal{M}_{a}$ on $L^{p}(\gamma)$, we obtain

$$
\left\|I_{a}^{\beta}(f)\right\|_{L^{q}(\gamma)}^{q} \lesssim\|f\|_{L^{p}(\gamma)}^{\beta p q} \int_{\mathbb{R}^{n}}\left[\mathcal{M}_{a}(f)(x)\right]^{q(1-\beta p)} d \gamma(x)=\left\|\mathcal{M}_{a}\right\|_{L^{p}(\gamma) \rightarrow L^{p}(\gamma)}^{p}\|f\|_{L^{p}(\gamma)}^{q},
$$

which implies that $I_{a}^{\beta}$ is bounded from $L^{p}(\gamma)$ to $L^{q}(\gamma)$.

Again using (3.2), (3.3) and the weak $(1,1)$ property of $\mathcal{M}_{a}$, we obtain that there exists a positive constant $C$ such that for all $\lambda>0$,

$$
\begin{aligned}
\gamma\left(\left\{x \in \mathbb{R}^{n}:\left|I_{a}^{\beta}(f)(x)\right|>\lambda\right\}\right) & =\gamma\left(\left\{x \in \mathbb{R}^{n}: C\|f\|_{L^{1}(\gamma)}^{\beta}\left[\mathcal{M}_{a}(f)(x)\right]^{1-\beta}>\lambda\right\}\right) \\
& \lesssim\left\|\mathcal{M}_{a}\right\|_{L^{1}(\gamma) \rightarrow L^{1, \infty}(\gamma)}\left(\frac{\|f\|_{L^{1}(\gamma)}}{\lambda}\right)^{1 /(1-\beta)},
\end{aligned}
$$

which implies that $I_{a}^{\beta}$ is bounded from $L^{1}(\gamma)$ to $L^{1 /(1-\beta), \infty}(\gamma)$. This finishes the proof of Theorem 3.1. 
Applying Theorem 3.1, we easily deduce the following conclusion.

Corollary 3.1 Let $a \in(0, \infty), \beta \in(0,1), 1<p<q<\infty$ and $1 / q=1 / p-\beta$. Then

(i) $\mathcal{M}_{a}^{\beta}$ is bounded from $L^{p}(\gamma)$ to $L^{q}(\gamma)$ and from $L^{1}(\gamma)$ to $L^{1 /(1-\beta), \infty}(\gamma)$;

(ii) $\mathcal{M}_{a}^{\beta}$ is bounded from $L^{1 / \beta}(\gamma)$ to $L^{\infty}(\gamma)$.

Proof. For all $x \in \mathbb{R}^{n}$ and $B \in \mathcal{B}_{a}(x)$, by (2.4), we obtain $B \subset B(x, 2 a(a+1) m(x))$. By this, (2.2) and (2.3), we further obtain

$$
\begin{aligned}
\mathcal{M}_{a}^{\beta}(f)(x) & \sim \sup _{B \in \mathcal{B}_{a}(x)} \frac{1}{\left[e^{\left.-\left|c_{B}\right|^{2} r_{B}^{n}\right]^{1-\beta}} \int_{B}|f(y)| d \gamma(y)\right.} \\
& \lesssim \sup _{B \in \mathcal{B}_{a}(x)} \int_{B} \frac{|f(y)|}{\left[e^{-|x|^{2}}|x-y|^{n}\right]^{1-\beta}} d \gamma(y) \\
& \lesssim \int_{B(x, 2 a(a+1) m(x))} \frac{|f(y)|}{[V(x, y)]^{1-\beta}} d \gamma(y)=I_{2 a(a+1)}^{\beta}(f)(x),
\end{aligned}
$$

which together with Theorem 3.1 implies (i). Property (ii) follows from Hölder's inequality. This finishes the proof of Corollary 3.1.

Applying Theorem 3.1, we now consider the end-point boundedness of $I_{a}^{\beta}$.

Theorem 3.2 Let $a \in(0, \infty), \beta \in(0,1), 1<p<q<\infty$ and $1 / q=1 / p-\beta$. Then

(i) both $I_{a}^{\beta}$ and $\widetilde{I}_{a}^{\beta}$ are bounded from $H^{1}(\gamma)$ to $L^{1 /(1-\beta)}(\gamma)$;

(ii) both $I_{a}^{\beta}$ and $\widetilde{I}_{a}^{\beta}$ are bounded from $\left\{f \in L^{1 / \beta}(\gamma): f \geq 0\right\}$ to $\operatorname{BLO}_{a}(\gamma)$;

(iii) both $I_{a}^{\beta}$ and $\widetilde{I}_{a}^{\beta}$ are bounded from $L^{1 / \beta}(\gamma)$ to $\operatorname{BMO}(\gamma)$.

Proof. We only prove the results of this theorem for the operator $I_{a}^{\beta}$, since the proof for $\widetilde{I}_{a}^{\beta}$ is similar but simpler.

To prove (i), first assume that $g$ is a $(1,2 /(1+\beta))$ atom supported on $B \in \mathcal{B}_{1}$ and show that $\left\|I_{a}^{\beta}(g)\right\|_{L^{1 /(1-\beta)}(\gamma)} \lesssim 1$. To this end, we write

$$
\int_{\mathbb{R}^{n}}\left|I_{a}^{\beta}(g)(x)\right|^{1 /(1-\beta)} d \gamma(x)=\int_{2 B}\left|I_{a}^{\beta}(g)(x)\right|^{1 /(1-\beta)} d \gamma(x)+\int_{(2 B)^{\complement}} \cdots \equiv \mathrm{Y}_{1}+\mathrm{Y}_{2}
$$

where and in what follows, $E^{\complement} \equiv \mathbb{R}^{n} \backslash E$ for any set $E \subset \mathbb{R}^{n}$.

By Hölder's inequality and (2.1) together with the fact $I_{a}^{\beta}$ is bounded from $L^{2 /(1+\beta)}(\gamma)$ to $L^{2 /(1-\beta)}(\gamma)$ (see Theorem 3.1), we obtain

$$
\begin{aligned}
\mathrm{Y}_{1} & \leq\left[\int_{2 B}\left|I_{a}^{\beta}(g)(x)\right|^{2 /(1-\beta)} d \gamma(x)\right]^{1 / 2}[\gamma(2 B)]^{1 / 2} \\
& \leq\left[\sigma_{1,2}^{*}\right]^{1 / 2}\left\|I_{a}^{\beta}\right\|_{L^{2 /(1+\beta)}(\gamma) \rightarrow L^{2 /(1-\beta)}(\gamma)}^{1 /(1-\beta)}\left[\int_{B}|g(x)|^{2 /(1+\beta)} d \gamma(x)\right]^{(1+\beta) /(2-2 \beta)}[\gamma(B)]^{1 / 2} \lesssim 1
\end{aligned}
$$


Now we estimate $\mathrm{Y}_{2}$. For any $x \notin 2 B$ satisfying $I_{a}^{\beta}(g)(x) \neq 0$, by (1.3) and supp $g \subset B$, there exists $w \in B \cap B(x, a m(x))$, which combined with (2.4) and $B \in \mathcal{B}_{1}$ yields

$$
(2 a+2)^{-1} m(x) \leq m\left(c_{B}\right) \leq(2 a+2) m(x) .
$$

For such an $x$, by (3.5) and the triangular inequality together with $r_{B} \leq m\left(c_{B}\right)$, we obtain $B \subset B(x,(5 a+4) m(x))$ and $x \in B\left(c_{B}, a^{*} m\left(c_{B}\right)\right)$ with $a^{*} \equiv 2 a(a+1)+1$. Thus,

$$
\mathrm{Y}_{2}=\int_{B\left(c_{B}, a^{*} m\left(c_{B}\right)\right) \cap(2 B)^{\complement}}\left|I_{a}^{\gamma}(g)(x)\right|^{1 /(1-\beta)} d \gamma(x) .
$$

For all $x \in B\left(c_{B}, a^{*} m\left(c_{B}\right)\right) \cap(2 B)^{\complement}$ satisfying $I_{a}^{\beta}(g)(x) \neq 0$, by the facts $\int_{B} g(x) d \gamma(x)=0$ and $B \subset B(x,(5 a+4) m(x))$, we write

$$
\begin{aligned}
\left|I_{a}^{\beta}(g)(x)\right| \leq & \left|\int_{B(x,(5 a+4) m(x))} g(y)\left[\frac{1}{[V(x, y)]^{1-\beta}}-\frac{1}{\left[V\left(x, c_{B}\right)\right]^{1-\beta}}\right] d \gamma(y)\right| \\
& +\left|\int_{B(x,(5 a+4) m(x)) \backslash B(x, a m(x))} \frac{g(y)}{[V(x, y)]^{1-\beta}} d \gamma(y)\right| \equiv \mathrm{I}_{1}+\mathrm{I}_{2} .
\end{aligned}
$$

Since $x \in B\left(c_{B}, a^{*} m\left(c_{B}\right)\right)$, then by $(2.2)$,

$$
e^{-\left|c_{B}\right|^{2}} \sim e^{-|x|^{2}}
$$

This combined with (3.5) yields

$$
\mathrm{I}_{2} \leq \frac{\|g\|_{L^{1}(\gamma)}}{[\gamma(B(x, a m(x)))]^{1-\beta}} \lesssim\left(e^{-\left|c_{B}\right|^{2}}\left[m\left(c_{B}\right)\right]^{n}\right)^{\beta-1} .
$$

To estimate $\mathrm{I}_{1}$, notice that for any $i \in\{1, \cdots, n\}$, a direct calculation yields that the $i$-th partial derivative of $V(x, y)$,

$$
\frac{\partial V(x, y)}{\partial y_{i}}=\pi^{-n / 2} \frac{x_{i}-y_{i}}{|x-y|^{2-n}} \int_{S^{n-1}} e^{-|| x-y\left|\xi^{\prime}+x\right|^{2}} d \sigma\left(\xi^{\prime}\right),
$$

where and in what follows, $S^{n-1} \equiv\left\{x \in \mathbb{R}^{n}:|x|=1\right\}$ and $d \sigma$ denotes the Lebesgue measure on the unite sphere $S^{n-1}$.

Applying (3.8) and the mean value theorem, we have

$$
\begin{aligned}
\mathrm{J} & \equiv\left|\frac{1}{[V(x, y)]^{1-\beta}}-\frac{1}{\left[V\left(x, c_{B}\right)\right]^{1-\beta}}\right| \\
& \leq \frac{(1-\beta) \pi^{-n / 2}\left|y-c_{B}\right|}{[V(x, y)]^{1-\beta}\left[V\left(x, c_{B}\right)\right]^{1-\beta}} \sup _{\substack{\theta \in(0,1) \\
z=\theta y+(1-\theta) c_{B}}}[V(x, z)]^{-\beta}|x-z|^{n-1} \int_{S^{n-1}} e^{-|| x-z\left|\xi^{\prime}+x\right|^{2}} d \sigma\left(\xi^{\prime}\right) .
\end{aligned}
$$

For any $x \notin 2 B, y \in B \subset B(x,(5 a+4) m(x))$ and $z=\theta y+(1-\theta) c_{B}$ with $\theta \in(0,1)$, by (2.3), we have $|x-y| \sim\left|x-c_{B}\right| \sim|x-z|$ and

$$
V(x, z) \sim V(x, y) \sim V\left(x, c_{B}\right) \sim e^{-\left|c_{B}\right|^{2}}\left|x-c_{B}\right|^{n} .
$$


Moreover, by (3.5), the facts that $\left|c_{B}\right| m\left(c_{B}\right) \leq 1$ and that $w \in B \cap B(x, a m(x))$, we have

$$
\begin{aligned}
|x||x-z| & \leq\left(|x-w|+\left|w-c_{B}\right|+\left|c_{B}\right|\right)\left(|x-w|+\left|w-c_{B}\right|+\left|c_{B}-z\right|\right) \\
& \leq\left(\operatorname{am}(x)+r_{B}+\left|c_{B}\right|\right)\left(\operatorname{am}(x)+2 r_{B}\right) \lesssim 1 .
\end{aligned}
$$

Combining (3.7), (3.9) and (3.10) yields

$$
\mathrm{J} \lesssim \frac{\left|y-c_{B}\right|\left|x-c_{B}\right|^{n-1} e^{-|x|^{2}}}{\left[e^{-\left|c_{B}\right|^{2}}\left|x-c_{B}\right|^{n}\right]^{2-\beta}} \lesssim \frac{\left|y-c_{B}\right| e^{\left|c_{B}\right|^{2}(1-\beta)}}{\left|x-c_{B}\right|^{n(1-\beta)+1}} .
$$

By the estimate of J, Hölder's inequality, (2.2) and (2.3), we obtain

$$
\begin{aligned}
\mathrm{I}_{1} & \lesssim \frac{e^{\left|c_{B}\right|^{2}(1-\beta)}}{\left|x-c_{B}\right|^{n(1-\beta)+1}} \int_{B}\left|y-c_{B}\right||g(y)| d \gamma(y) \\
& \lesssim \frac{e^{\left|c_{B}\right|^{2}(1-\beta)}}{\left|x-c_{B}\right|^{n(1-\beta)+1}}\|g\|_{L^{2 /(1+\beta)}(\gamma)}\left\{\int_{B}\left|y-c_{B}\right|^{2 /(1-\beta)} d \gamma(y)\right\}^{(1-\beta) / 2} \\
& \lesssim \frac{e^{\left|c_{B}\right|^{2}(1-\beta)}}{\left|x-c_{B}\right|^{n(1-\beta)+1}}[\gamma(B)]^{(\beta-1) / 2} r_{B}^{1+n(1-\beta) / 2} e^{-\left|c_{B}\right|^{2}(1-\beta) / 2} \lesssim r_{B} \frac{e^{\left|c_{B}\right|^{2}(1-\beta)}}{\left|x-c_{B}\right|^{n(1-\beta)+1}}
\end{aligned}
$$

Inserting the estimates of $\mathrm{I}_{1}$ and $\mathrm{I}_{2}$ into (3.6) yields

$$
\mathrm{Y}_{2} \lesssim \int_{B\left(c_{B}, a^{*} m\left(c_{B}\right)\right) \cap(2 B)^{\complement}}\left[r_{B}^{1 /(1-\beta)} \frac{e^{\left|c_{B}\right|^{2}}}{\left|x-c_{B}\right|^{n+1 /(1-\beta)}}+e^{\left|c_{B}\right|^{2}} m\left(c_{B}\right)^{-n}\right] d \gamma(x) \lesssim 1 .
$$

The estimates of $\mathrm{Y}_{1}$ and $\mathrm{Y}_{2}$ together with (3.4) imply $\left\|I_{a}^{\beta}(g)\right\|_{L^{1 /(1-\beta)}(\gamma)} \lesssim 1$.

If $g$ is the constant function 1 , then by (2.2) and (2.3), we have

$$
\left|I_{a}^{\beta}(g)(x)\right| \sim \int_{B(x, a m(x))} \frac{1}{\left[e^{-|x|^{2}}|x-y|^{n}\right]^{1-\beta}} d \gamma(y) \lesssim \int_{0}^{a} s^{n-1-n(1-\beta)} d r \lesssim 1,
$$

which together with $\gamma\left(\mathbb{R}^{n}\right)=1$ further implies that $\left\|I_{a}^{\beta}(g)\right\|_{L^{1 /(1-\beta)}(\gamma)} \lesssim 1$. Therefore, $I_{a}^{\beta}$ maps all $(1,2 /(1+\beta))$ atoms into uniformly bounded elements of $L^{1 /(1-\beta)}(\gamma)$.

For any $f \in H^{1}(\gamma)$, we can write $f=\sum_{j=1}^{\infty} \lambda_{j} a_{j}$, where $\left\{a_{j}\right\}_{j=1}^{\infty}$ are $(1,2 /(1+\beta))$ atoms and $\sum_{j=1}^{\infty}\left|\lambda_{j}\right| \sim\|f\|_{H^{1}(\gamma)}$. By the facts $H^{1}(\gamma) \subset L^{1}(\gamma)$ and the boundedness of $I_{a}^{\beta}$ from $L^{1}(\gamma)$ to $L^{1 /(1-\beta), \infty}(\gamma)$, we obtain that $I_{a}^{\beta}(f)$ is a well-defined $L^{1 /(1-\beta), \infty}(\gamma)$ function. Moreover, for almost all $x \in \mathbb{R}^{n}$,

$$
I_{a}^{\beta}(f)(x)=\sum_{j=1}^{\infty} \lambda_{j} I_{a}^{\beta}\left(a_{j}\right)(x)
$$

see, for example, the proof of $(6.7 .9)$ in $\left[8\right.$, p. 95]. Then taking $L^{1 /(1-\beta)}(\gamma)$ norms on both sides of (3.11) yields that $I_{a}^{\beta}$ is bounded from $H^{1}(\gamma)$ to $L^{1 /(1-\beta)}(\gamma)$. Hence, (i) holds. 
Now we show (ii). For all $f \in L^{1 / \beta}(\gamma)$, by (2.2) through (2.4) together with Hölder's inequality, we obtain

$$
\begin{aligned}
\left\|I_{a}^{\beta}(f)\right\|_{L^{1}(\gamma)} & =\left|\int_{\mathbb{R}^{n}} \int_{B(x, a m(x))} \frac{f(y)}{[V(x, y)]^{1-\beta}} d \gamma(y) d \gamma(x)\right| \\
& \lesssim \int_{\mathbb{R}^{n}}\left[\int_{|x-y|<a(a+1) m(y)} \frac{e^{-|x|^{2}}}{\left[e^{\left.-|x|^{2}|x-y|^{n}\right]^{1-\beta}}\right.} d x\right]|f(y)| d \gamma(y) \\
& \lesssim\|f\|_{L^{1}(\gamma)} \lesssim\|f\|_{L^{1 / \beta}(\gamma)} .
\end{aligned}
$$

Thus, to finish the proof of (ii), by (3.12) and (2.9), it suffices to show that there exists a positive constant $C$ such that for all $f \geq 0$ and $B \in \mathcal{B}_{a}$,

$$
\frac{1}{\gamma(B)} \int_{B} I_{a}^{\beta}(f)(y) d \gamma(y) \leq C\|f\|_{L^{1 / \beta}(\gamma)}+\underset{x \in B}{\operatorname{essinf}} I_{a}^{\beta}(f)(x) .
$$

To see (3.13), we decompose $f$ into $f=f \chi_{3 B}+f \chi_{(3 B)^{\complement}}$. Choose $p \in(1,1 / \beta)$ and $1 / q=1 / p-\beta$. Using Hölder's inequality and (2.1) together with Theorem 3.1, we obtain

$$
\begin{aligned}
\frac{1}{\gamma(B)} \int_{B} I_{a}^{\beta}\left(f \chi_{3 B}\right)(y) d \gamma(y) & \leq\left\{\frac{1}{\gamma(B)} \int_{B}\left|I_{a}^{\beta}\left(f \chi_{3 B}\right)(y)\right|^{q} d \gamma(y)\right\}^{1 / q} \\
& \leq\left\|I_{a}^{\beta}\right\|_{L^{p}(\gamma) \rightarrow L^{q}(\gamma)}[\gamma(B)]^{-1 / q}\left\|f \chi_{3 B}\right\|_{L^{p}(\gamma)} \\
& \leq\left[\sigma_{a, 3}^{*}\right]^{1 / q}\left\|I_{a}^{\beta}\right\|_{L^{p}(\gamma) \rightarrow L^{q}(\gamma)}\|f\|_{L^{1 / \beta}(\gamma)} .
\end{aligned}
$$

By this and the linearity of $I_{a}^{\beta}$, we know that to obtain (3.13), it is enough to show that for all $y \in B$ and $x \in B$,

$$
I_{a}^{\beta}\left(f \chi_{(3 B)^{\mathrm{C}}}\right)(y) \leq C\|f\|_{L^{1 / \beta}(\gamma)}+I_{a}^{\beta}(f)(x) .
$$

If $I_{a}^{\beta}(f)(x)=\infty$, then (3.14) holds trivially. Assume now that $I_{a}^{\beta}(f)(x)<\infty$. Notice that $f \geq 0$, and hence $I_{a}^{\beta}(f)(x)$ is finite. Thus,

$$
\begin{aligned}
& I_{a}^{\beta}\left(f \chi_{(3 B)^{\mathrm{C}}}\right)(y)-I_{a}^{\beta}(f)(x) \\
& \quad \leq \int_{B(y, a m(y))} \frac{f(z) \chi_{(3 B)^{\mathrm{C}}}(z)}{[V(y, z)]^{1-\beta}} d \gamma(z)-\int_{B(x, a m(x))} \frac{f(z) \chi_{(3 B)^{\complement}}(z)}{[V(x, z)]^{1-\beta}} d \gamma(z) \equiv \mathrm{Y} .
\end{aligned}
$$

If $B \in \mathcal{B}_{a}, x \in B$ and $y \in B$, then by (2.4), we obtain that for all $z \in B(y, a m(y))$,

$$
|z-x| \leq|z-y|+|y-x|<a m(y)+2 r_{B} \leq a(a+1) m\left(c_{B}\right)+2 a m\left(c_{B}\right) \leq \widetilde{a} m(x),
$$

where $\widetilde{a} \equiv a(a+1)(a+3)$. Thus, $B(y, a m(y)) \subset B(x, \widetilde{a} m(x))$. It follows that

$$
\mathrm{Y} \leq \int_{B(x, \widetilde{a} m(x))} \frac{f(z) \chi_{(3 B)^{\mathrm{C}}}(z)}{[V(y, z)]^{1-\beta}} d \gamma(z)-\int_{B(x, a m(x))} \frac{f(z) \chi_{(3 B)^{\mathrm{C}}}(z)}{[V(x, z)]^{1-\beta}} d \gamma(z)
$$




$$
\begin{aligned}
= & \int_{B(x, \widetilde{a} m(x))} f(z) \chi_{(3 B)^{\complement}}(z)\left[\frac{1}{[V(y, z)]^{1-\beta}}-\frac{1}{[V(x, z)]^{1-\beta}}\right] d \gamma(z) \\
& +\int_{B(x, \widetilde{a} m(x)) \backslash B(x, a m(x))} \frac{f(z) \chi_{(3 B)}{ }^{\complement}(z)}{[V(x, z)]^{1-\beta}} d \gamma(z) \equiv \mathrm{J}_{1}+\mathrm{J}_{2} .
\end{aligned}
$$

Using Hölder's inequality and (2.1) yields

$$
\mathrm{J}_{2} \leq \frac{1}{[\gamma(B(x, a m(x)))]^{1-\beta}} \int_{B(x, \widetilde{a} m(x))} f(z) d \gamma(z) \lesssim\|f\|_{L^{1 / \beta}(\gamma)} .
$$

Now we estimate $\mathrm{J}_{1}$. Notice that for all $i \in\{1, \cdots, n\}$, a simple calculation yields

$$
\begin{aligned}
\frac{\partial V(y, z)}{\partial y_{i}}= & \pi^{-n / 2} \frac{y_{i}-z_{i}}{|y-z|^{2-n}} \int_{S^{n-1}} e^{-|| y-z\left|\xi^{\prime}+z\right|^{2}} d \sigma\left(\xi^{\prime}\right) \\
& -2 \pi^{-n / 2} \int_{|\xi-y|<|y-z|}\left(\xi_{i}-y_{i}\right) e^{-|\xi|^{2}} d \xi .
\end{aligned}
$$

This together with an argument similar to the estimates of $\mathrm{J}$ and $\mathrm{I}_{1}$ implies that

$$
\begin{aligned}
\mathrm{J}_{1} \leq & \int_{B(x, \widetilde{a} m(x))} \frac{(1-\beta) f(z) \chi_{(3 B)^{\mathrm{C}}}(z)|y-x|}{[V(y, z)]^{1-\beta}[V(x, z)]^{1-\beta}} \sup _{\substack{\theta \in(0,1) \\
w=\theta x+(1-\theta) y}}\left|[V(w, z)]^{-\beta} \frac{\partial V(w, z)}{\partial w_{i}}\right| d \gamma(z) \\
\lesssim & \frac{f(z) \chi_{B} \int_{B(3 B)^{\mathrm{C}}(z)}}{} \sup _{\substack{\theta \in(0,1) \\
w=\theta x+(1-\theta) y}}\left\{|w-z|^{n-1} \int_{S^{n-1}} e^{-|| w-z\left|\xi^{\prime}+z\right|^{2}} d \sigma\left(\xi^{\prime}\right)\right. \\
& \left.+\int_{|\xi-w|<|w-z|}|\xi-w| e^{-|\xi|^{2}} d \xi\right\} d \gamma(z) \\
\lesssim & r_{B} \int_{B(x, \widetilde{a} m(x))} \frac{f(z) \chi_{(3 B)^{\mathrm{C}}}(z)}{\left[e^{\left.-|x|^{2}|x-z|^{2}\right]^{2-\beta}} e^{-|z|^{2}}|x-z|^{n-1} d \gamma(z) \lesssim\|f\|_{L^{1 / \beta}(\gamma)},\right.}
\end{aligned}
$$

where in the penultimate inequality, we used the fact that $|w-z| \sim|x-z| \leq \widetilde{a}$ and (2.2) together with $|\xi-z| \lesssim m(z)$. Combining the estimates of $\mathrm{J}_{1}$ and $\mathrm{J}_{2}$ yields the desired estimate of $\mathrm{Y}$, and hence (3.14). Thus, (ii) holds.

Finally we prove (iii). For any $f \in L^{1 / \beta}(\gamma)$, we decompose $f=f^{+}-f^{-}$, where $f^{+} \equiv \max \{f, 0\}$ and $f^{-} \equiv \min \{f, 0\}$. From (2.10) and (ii) of this theorem, it follows that

$$
\begin{aligned}
\left\|I_{a}^{\beta}(f)\right\|_{\mathrm{BMO}(\gamma)} & \leq\left\|I_{a}^{\beta}\left(f^{+}\right)\right\|_{\mathrm{BMO}(\gamma)}+\left\|I_{a}^{\beta}\left(f^{-}\right)\right\|_{\mathrm{BMO}(\gamma)} \\
& \lesssim\left\|I_{a}^{\beta}\left(f^{+}\right)\right\|_{\mathrm{BLO}_{a}(\gamma)}+\left\|I_{a}^{\beta}\left(f^{-}\right)\right\|_{\mathrm{BLO}_{a}(\gamma)} \lesssim\|f\|_{L^{1 / \beta}(\gamma)} .
\end{aligned}
$$

Thus (iii) holds, which completes the proof of Theorem 3.2.

\section{Proofs of Theorems 1.1 through 1.3}

In this section, by applying Theorem 3.1 and the geometry properties listed in Section 2 together with some ideas used in the Euclidean case (see, for example, $[12,3,15,8]$ ), we prove Theorems 1.1 and 1.2. 
We begin with two technical lemmas. For any $a \in(0, \infty), \beta \in(0,1)$ and $b \in \operatorname{BMO}(\gamma)$, define the following auxiliary operator $T_{a}^{\beta}(b ; \cdot)$ by setting, for all locally integrable functions $f$ and $x \in \mathbb{R}^{n}$,

$$
T_{a}^{\beta}(b ; f)(x) \equiv \int_{B(x, a m(x))} \frac{|b(x)-b(y)||f(y)|}{\left[e^{\left.-|y|^{2}|x-y|^{n}\right]^{(1-\beta)}}\right.} d \gamma(y) .
$$

Correspondingly, we introduce another auxiliary operator $\widetilde{T_{a}^{\beta}(b ; \cdot)}$, which is in fact a smooth version of $T_{a}^{\beta}(b ; \cdot)$. Precisely, let $\phi$ be a radial function in $C_{c}^{\infty}(\mathbb{R})$ such that $0 \leq \phi \leq 1, \phi(t) \equiv 1$ when $|t|<1, \phi(t) \equiv 0$ when $|t| \geq 2$; moreover, there exists a positive constant $C$ such that $\left|\phi^{\prime}(t)\right| \leq C /|t|$ for all $t \in \mathbb{R}$. For any $x, y \in \mathbb{R}^{n}$, we set

$$
\phi_{x, y} \equiv \phi\left(\frac{|x-y|}{a(a+1) m(y)}\right) \text {. }
$$

Define

$$
\widetilde{T_{a}^{\beta}(b ; f)}(x) \equiv \int_{\mathbb{R}^{n}} \frac{\phi_{x, y}|b(x)-b(y)||f(y)|}{\left[e^{\left.-|y|^{2}|x-y|^{n}\right]^{(1-\beta)}}\right.} d \gamma(y) .
$$

By (2.4) and the support condition of $\phi$, it is not difficult to see that for all locally integrable functions $f$ and $x \in \mathbb{R}^{n}$,

$$
T_{a}^{\beta}(b ; f)(x) \leq \widetilde{T_{a}^{\beta}(b ; f)}(x) \leq T_{\widetilde{C}_{a}}^{\beta}(b ; f)(x),
$$

where $\widetilde{C}_{a} \equiv 2 a(a+1)\left(2 a^{2}+2 a+1\right)$. It follows from (2.2) that for all $x \in \mathbb{R}^{n}$,

$$
\left|\left[b, \widetilde{I}_{a}^{\beta}\right](f)(x)\right| \leq \widehat{\left[b, I_{a}^{\beta}\right]}(f)(x) \sim T_{a}^{\beta}(b ; f)(x) .
$$

The following lemma is used in the proof of Theorem 1.1 due to the extra term $\|\cdot\|_{L^{1}(\gamma)}$ appearing in (2.7), comparing to the classical case of Euclidean spaces (see, for example, $[21$, p. 148]).

Lemma 4.1 Let $a \in(0, \infty), \beta \in(0,1), p \in(1,1 / \beta)$ and $b \in \operatorname{BMO}(\gamma)$. Then both $T_{a}^{\beta}(b ; \cdot)$ and $\widetilde{\left[b, I_{a}^{\beta}\right]}$ are bounded from $L^{p}(\gamma)$ to $L^{1}(\gamma)$ with norm at most a constant multiple of $\|b\|_{*}$.

Proof. By (4.4), we only need to consider $T_{a}^{\beta}(b ; \cdot)$. To this end, let $\left\{B_{j}\right\}_{j \in \mathbb{N}}$ be the sequence of balls as in Lemma 2.1. If $x \in B_{j}$, then by (2.4), we obtain that for all $y \in B(x, a m(x)),\left|y-x_{j}\right|<(2 a+1) m\left(x_{j}\right)$. Thus, we have $B(x, a m(x)) \subset \frac{1}{\kappa}(2 a+1) B_{j}$, where $\kappa=\frac{1}{20}$ is as in Lemma 2.1. Set $\widetilde{a} \equiv \frac{1}{\kappa}(2 a+1)$. Then for any $z \in \widetilde{a} B_{j}$, again using (2.4), we obtain $|z-x|<4(a+1) m(x)$, and hence $\widetilde{a} B_{j} \subset B(x, 4(a+1) m(x))$. From these facts together with $\mathbb{R}^{n} \subset \cup_{j \in \mathbb{N}} B_{j}$, it follows that

$$
\left\|T_{a}^{\beta}(b ; f)\right\|_{L^{1}(\gamma)} \lesssim \sum_{j \in \mathbb{N}} \int_{B_{j}} \int_{B(x, a m(x))} \frac{|b(x)-b(y)||f(y)|}{[V(x, y)]^{1-\beta}} d \gamma(y) d \gamma(x)
$$


Characterizations of BMO Associated with Gauss Measures

$$
\begin{aligned}
\lesssim & \sum_{j \in \mathbb{N}} \int_{B_{j}} \int_{\widetilde{a} B_{j}} \frac{|b(x)-b(y)||f(y)|}{[V(x, y)]^{1-\beta}} d \gamma(y) d \gamma(x) \\
\lesssim & \sum_{j \in \mathbb{N}} \int_{B_{j}}\left|b(x)-b_{\widetilde{a} B_{j}}\right| I_{4(a+1)}^{\beta}(|f|)(x) d \gamma(x) \\
& +\sum_{j \in \mathbb{N}} \int_{B_{j}} I_{4(a+1)}^{\beta}\left(\left|b-b_{\widetilde{a} B_{j}}\right||f| \chi_{\widetilde{a} B_{j}}\right)(x) d \gamma(x) \equiv \mathrm{I}+\mathrm{J} .
\end{aligned}
$$

Choose $q \in(0, \infty)$ satisfying that $1 / q=1 / p-\beta$. Applying Hölder's inequality for integrals and series, Lemma 2.1, Theorem 3.1, (2.1), (2.8) and the fact $\gamma\left(\mathbb{R}^{n}\right)=1$, we obtain

$$
\begin{aligned}
\mathrm{I} & \leq \sum_{j \in \mathbb{N}}\left\{\int_{B_{j}}\left|b(x)-b_{\widetilde{a} B_{j}}\right|^{q^{\prime}} d \gamma(x)\right\}^{1 / q^{\prime}}\left\{\int_{B_{j}}\left|I_{4(a+1)}^{\beta}(|f|)(x)\right|^{q} d \gamma(x)\right\}^{1 / q} \\
& \lesssim\left\{\sum_{j \in \mathbb{N}} \int_{B_{j}}\left|b(x)-b_{\widetilde{a} B_{j}}\right|^{q^{\prime}} d \gamma(x)\right\}^{1 / q^{\prime}}\left\|I_{4(a+1)}^{\beta}(|f|)\right\|_{L^{q}(\gamma)} \lesssim\|b\|_{*}\|f\|_{L^{p}(\gamma)} .
\end{aligned}
$$

Now we estimate J. Choose $s \in(1, p)$ and $r \in(1, q)$ such that $1 / r=1 / s-\beta$. Then by Hölder's inequality and Theorem 3.1,

$$
\begin{aligned}
\mathrm{J} & \leq \sum_{j \in \mathbb{N}}\left[\gamma\left(B_{j}\right)\right]^{1 / r^{\prime}}\left\{\int_{B_{j}}\left[I_{4(a+1)}^{\beta}\left(\left|b-b_{\widetilde{a} B_{j}}\right||f| \chi_{\widetilde{a} B_{j}}\right)(x)\right]^{r} d \gamma(x)\right\}^{1 / r} \\
& \lesssim \sum_{j \in \mathbb{N}}\left[\gamma\left(B_{j}\right)\right]^{1 / r^{\prime}}\left\{\int_{\widetilde{a} B_{j}}\left[\left|b(x)-b_{\widetilde{a} B_{j}}\right||f(x)|\right]^{s} d \gamma(x)\right\}^{1 / s} .
\end{aligned}
$$

Using Hölder's inequality again yields that the last quantity above is bounded by

$$
\sum_{j \in \mathbb{N}}\left[\gamma\left(B_{j}\right)\right]^{1 / r^{\prime}}\left\{\int_{\widetilde{a} B_{j}}\left|b(x)-b_{\widetilde{a} B_{j}}\right|^{p s /(p-s)} d \gamma(x)\right\}^{1 / s-1 / p}\left\{\int_{\widetilde{a} B_{j}}|f(x)|^{p} d \gamma(x)\right\}^{1 / p} .
$$

Then by (2.1), Hölder's inequality for series, (2.8), Lemma 2.1 and $\gamma\left(\mathbb{R}^{n}\right)=1$, we finally obtain

$$
\begin{aligned}
\mathrm{J} & \lesssim\|b\|_{*} \sum_{j \in \mathbb{N}}\left[\gamma\left(B_{j}\right)\right]^{1 / r^{\prime}+1 / s-1 / p}\left\{\int_{\widetilde{a} B_{j}}|f(x)|^{p} d \gamma(x)\right\}^{1 / p} \\
& \lesssim\|b\|_{*}\|f\|_{L^{p}(\gamma)}\left\{\sum_{j \in \mathbb{N}}\left[\gamma\left(B_{j}\right)\right]^{p^{\prime}\left(1 / r^{\prime}+1 / s-1 / p\right)}\right\}^{1 / p^{\prime}} \lesssim\|b\|_{*}\|f\|_{L^{p}(\gamma)},
\end{aligned}
$$

where in the last step we use $p^{\prime}\left(1 / r^{\prime}+1 / s-1 / p\right)=1+p^{\prime} \beta$ and $\sum_{j \in \mathbb{N}}\left[\gamma\left(B_{j}\right)\right]^{1+p^{\prime} \beta} \lesssim 1$. Combining the estimates of I and II yields the desired result for $T_{a}^{\beta}(b ; \cdot)$, which completes the proof of Lemma 4.1. 
For the Euclidean version of the following Lemma 4.2, see [12, Lemma 11] or [23, p. 418].

Lemma 4.2 Let $a \in(0, \infty), \beta \in(0,1), 1<p<q<\infty$ and $1 / q=1 / p-\beta$. Let $b \in \operatorname{BMO}(\gamma)$ and $\widehat{T_{a}^{\beta}(b ; f)}$ be as in (4.2). Then for any fixed $r, s \in(1, p)$, there exists a positive constant $C$ such that for all $f \in L_{c}^{\infty}(\gamma)$ and $x \in \mathbb{R}^{n}$,

$$
\left(\widetilde{T_{a}^{\beta}(b ; f)}\right)^{\sharp}(x) \leq C\|b\|_{*}\left\{\left[\mathcal{M}_{1}\left(\left[I_{C_{a}}^{\beta}(|f|)\right]^{r}\right)(x)\right]^{1 / r}+\left[\mathcal{M}_{C_{a}}^{\beta s}\left(|f|^{s}\right)(x)\right]^{1 / s}\right\},
$$

where $C_{a}$ is a sufficiently large positive constant depending only on $a,\left(\widetilde{T_{a}^{\beta}(b ; f)}\right)^{\sharp}$ is as in (2.6) with $f$ replaced by $\widetilde{T_{a}^{\beta}(b ; f)}, \mathcal{M}_{1}$ is as in $(2.5)$ with $a=1$ and $\mathcal{M}_{C_{a}}^{\beta s}$ is as in (1.5) with $\beta$ and a there replaced, respectively, by $\beta$ s and $C_{a}$.

Proof. Fix $x \in \mathbb{R}^{n}$. To show (4.5), for any fixed ball $B^{\prime} \in \mathcal{B}_{1}(x)$ and any $y \in B^{\prime}$, we write

$$
\begin{aligned}
& \left.\widetilde{T_{a}^{\beta}(b ; f}\right)(y) \leq\left|b(y)-b_{B^{\prime}}\right| \int_{\mathbb{R}^{n}} \frac{\phi_{y, z}|f(z)|}{\left[e^{-|z|^{2}}|y-z|^{n}\right]^{1-\beta}} d \gamma(z) \\
& +\int_{\mathbb{R}^{n}} \frac{\phi_{y, z}\left|b_{B^{\prime}}-b(z)\right||f(z)| \chi_{3 B^{\prime}}(z)}{\left[e^{-|z|^{2}}|y-z|^{n}\right]^{1-\beta}} d \gamma(z) \\
& +\int_{\mathbb{R}^{n}} \frac{\phi_{y, z}\left|b_{B^{\prime}}-b(z)\right||f(z)| \chi_{\left(3 B^{\prime}\right)^{\complement}}(z)}{\left[e^{-|z|^{2}}|y-z|^{n}\right]^{1-\beta}} d \gamma(z) \\
& \equiv \mathcal{J}_{1}^{B^{\prime}}(y)+\mathcal{J}_{2}^{B^{\prime}}(y)+\mathcal{J}_{3}^{B^{\prime}}(y) \text {. }
\end{aligned}
$$

For all $z \in \mathbb{R}^{n}$ satisfying $\phi_{y, z} \neq 0$, i.e, $|z-y|<2 a(a+1) m(z)$, by (2.2) and (2.3), we have,

$$
e^{-|z|^{2}}|z-y|^{n} \sim e^{-|y|^{2}}|z-y|^{n} \sim V(z, y) .
$$

This and the support condition of $\phi_{y, z}$ together with Hölder's inequality and (2.8) imply that

$$
\frac{1}{\gamma\left(B^{\prime}\right)} \int_{B^{\prime}} \mathcal{J}_{1}^{B^{\prime}}(y) d \gamma(y) \lesssim\|b\|_{*}\left[\mathcal{M}_{1}\left(\left[I_{C_{a}}^{\beta}(|f|)\right]^{r}\right)(x)\right]^{1 / r}
$$

for some positive constant $C_{a}$ which depends only on $a$.

Choose $\delta>1$ and $\kappa>1$ such that $\delta \kappa=s$. Since $1<\kappa<p<1 / \beta$, there exists $u>\kappa$ such that $1 / u=1 / \kappa-\beta$. By (4.6), Theorem 3.1, Hölder's inequality and $\delta \kappa=s$, we obtain that for sufficiently large positive number $C_{a}$,

$$
\begin{aligned}
\frac{1}{\gamma\left(B^{\prime}\right)} \int_{B^{\prime}} \mathcal{J}_{2}^{B^{\prime}}(y) d \gamma(y) & \lesssim\left\{\frac{1}{\gamma\left(B^{\prime}\right)} \int_{B^{\prime}}\left|I_{C_{a}}^{\beta}\left(\left|b_{B^{\prime}}-b\right||f| \chi_{3 B^{\prime}}\right)(y)\right|^{u} d \gamma(y)\right\}^{1 / u} \\
& \leq \frac{1}{\left[\gamma\left(B^{\prime}\right)\right]^{1 / u}}\left\{\int_{3 B^{\prime}}\left|b(y)-b_{B^{\prime}}\right|^{\kappa \delta^{\prime}} d \gamma(y)\right\}^{1 /\left(\kappa \delta^{\prime}\right)}\left\{\int_{3 B^{\prime}}|f(y)|^{s}\right\}^{1 / s} .
\end{aligned}
$$


Notice that the triangular inequality of $\|\cdot\|_{L^{\kappa \delta^{\prime}}(\gamma)}$ and (2.1) imply

$$
\begin{aligned}
\left\{\frac{1}{\gamma\left(3 B^{\prime}\right)} \int_{3 B^{\prime}}\left|b(y)-b_{B^{\prime}}\right|^{\kappa \delta^{\prime}} d \gamma(y)\right\}^{1 /\left(\kappa \delta^{\prime}\right)} & \leq\|b\|_{*}^{\mathcal{B}_{3}, \kappa \delta^{\prime}}+\left|b_{3 B^{\prime}}-b_{B^{\prime}}\right| \\
& \leq\|b\|_{*}^{\mathcal{B}_{3}, \kappa \delta^{\prime}}+\sigma_{1,3}^{*}\|b\|_{*}^{3,1} .
\end{aligned}
$$

This combined with (2.8) and (2.1) yields

$$
\frac{1}{\gamma\left(B^{\prime}\right)} \int_{B^{\prime}} \mathcal{J}_{2}^{B^{\prime}}(y) d \gamma(y) \lesssim\|b\|_{*}\left[\mathcal{M}_{3}^{\beta s}\left(|f|^{s}\right)(x)\right]^{1 / s}
$$

Let $y \in B^{\prime}$ and $z \notin 3 B^{\prime}$ satisfying that $\frac{\phi_{y, z}}{|y-z|^{n(1-\beta)}}-\frac{\phi_{c_{B^{\prime}}, z}}{\left|c_{B^{\prime}}-z\right|^{n(1-\beta)}} \neq 0$. Then we use (2.4) to obtain that $|z-y| \leq C_{a} m(y)$ for some large enough positive constant $C_{a}$ that depends only on $a$. From this, the mean value theorem, the definition of $\phi$ and the fact that $|z-y| \sim\left|z-c_{B^{\prime}}\right|$ for all $y \in B^{\prime}$ and $z \notin 3 B^{\prime}$, we easily deduce that

$$
\left|\frac{\phi_{y, z}}{|y-z|^{n(1-\beta)}}-\frac{\phi_{c_{B^{\prime}}, z}}{\left|c_{B^{\prime}}-z\right|^{n(1-\beta)}}\right| \lesssim \frac{\left|y-c_{B^{\prime}}\right|}{|y-z|^{n(1-\beta)+1}} \chi_{B\left(y, C_{a} m(y)\right)}(z),
$$

which combined with Hölder's inequality further implies that for all $y \in B^{\prime}$,

$$
\begin{aligned}
\left|\mathcal{J}_{3}^{B^{\prime}}(y)-\mathcal{J}_{3}^{B^{\prime}}\left(c_{B^{\prime}}\right)\right| & \lesssim \int_{\left(3 B^{\prime}\right)^{\complement} \cap B\left(y, C_{a} m(y)\right)} \frac{\left|b_{B^{\prime}}-b(z)\right||f(z)|\left|y-c_{B^{\prime}}\right|}{e^{-|z|^{2}(1-\beta)\left|z-c_{B^{\prime}}\right|^{1+n-n \beta}} d \gamma(z)} \\
\leq & \left\{r_{B^{\prime}} \int_{\left(3 B^{\prime}\right)^{\complement} \cap B\left(y, C_{a} m(y)\right)} \frac{\left|b_{B^{\prime}}-b(z)\right|^{s^{\prime}}}{\left.e^{-|z|^{2}\left|z-c_{B^{\prime}}\right|^{1+n}} d \gamma(z)\right\}^{1 / s^{\prime}}}\right. \\
& \times\left\{r_{B^{\prime}} \int_{\left(3 B^{\prime}\right)^{\complement} \cap B\left(y, C_{a} m(y)\right)} \frac{|f(z)|^{s}}{e^{-|z|^{2}(1-\beta s)}\left|z-c_{B^{\prime}}\right|^{1+n-n \beta s}} d \gamma(z)\right\}^{1 / s} \\
& \equiv \mathrm{I} \times \mathrm{J} .
\end{aligned}
$$

For all $z \in B\left(y, C_{a} m(y)\right)$, by $(2.2)$ and $y \in B^{\prime}$, we have $e^{-|z|^{2}} \sim e^{-|y|^{2}} \sim e^{-\left|c_{B^{\prime}}\right|^{2}}$. Thus,

$$
\mathrm{I} \lesssim \sum_{j=1}^{\infty}\left\{\frac{2^{-j}}{\gamma\left(2^{j} B^{\prime}\right)} \int_{\substack{|z-y|<C_{a} m(y) \\ 2^{j} B_{B^{\prime}}<\left|z-c_{B^{\prime}}\right| \leq 2^{j+1} r_{B^{\prime}}}}\left|b_{B^{\prime}}-b(z)\right|^{s^{\prime}} d \gamma(z)\right\}^{1 / s^{\prime}} .
$$

Notice that for $j \in \mathbb{N}$ satisfying $2^{j} r_{B^{\prime}}<\left|z-c_{B^{\prime}}\right|$ and $|z-y|<C_{a} m(y)$, we have

$$
2^{j} r_{B^{\prime}}<\left|z-c_{B^{\prime}}\right| \leq|z-y|+\left|y-c_{B^{\prime}}\right|<C_{a} m(y)+r_{B^{\prime}} \leq\left[C_{a}(a+1)+a\right] m\left(c_{B^{\prime}}\right),
$$

and hence, $2^{j+1} B^{\prime} \in \mathcal{B}_{C_{a}(a+1)+a}$. For simplicity, we set $a^{*} \equiv C_{a}(a+1)+a$. This, together with Minkowski's inequality, (2.1) and (2.8), implies that

$$
\mathrm{I} \lesssim \sum_{\left\{j \in \mathbb{N}: 2^{j}\right.} \sum_{\left.r_{B^{\prime}}<a^{*} m\left(c_{B^{\prime}}\right)\right\}} 2^{-j / s^{\prime}}\left(\left|b_{B^{\prime}}-b_{2 B^{\prime}}\right|+\cdots+\left|b_{2^{j} B^{\prime}}-b_{2^{j+1} B^{\prime}}\right|\right.
$$




$$
\begin{aligned}
& \left.+\left\{\frac{1}{\gamma\left(2^{j} B^{\prime}\right)} \int_{2^{j+1} B^{\prime}}\left|b_{2^{j+1} B^{\prime}}-b(z)\right|^{s^{\prime}} d \gamma(z)\right\}^{1 / s^{\prime}}\right) \\
\lesssim & \sum_{j=1}^{\infty} 2^{-j / s^{\prime}}\left(j\|b\|_{*}^{\mathcal{B}_{a^{*}}, 1}+\|b\|_{*}^{\mathcal{B}_{2 a^{*}}, s^{\prime}}\right) \lesssim\|b\|_{*} .
\end{aligned}
$$

Similarly to the estimate of I, we have

$$
\begin{aligned}
& \mathrm{J} \lesssim \sum_{j=1}^{\infty}\left\{\frac{2^{-j}}{\left[\gamma\left(2^{j} B^{\prime}\right)\right]^{1-\beta s}} \int_{2_{{ }^{j}{ }_{B^{\prime}}<\left|z-c_{B^{\prime}}\right| \leq 2^{j+1} r_{B^{\prime}}}|z-y|<a^{*} m(y)}|f(z)|^{s} d \gamma(z)\right\}^{1 / s} \\
& \lesssim \sum_{\left\{j \in \mathbb{N}: 2^{j} r_{B^{\prime}}<a^{*} m\left(c_{B^{\prime}}\right)\right\}}\left\{\frac{2^{-j}}{\left[\gamma\left(2^{j} B^{\prime}\right)\right]^{1-\beta s}} \int_{2^{j+1} B^{\prime}}|f(z)|^{s} d \gamma(z)\right\}^{1 / s} \lesssim\left[\mathcal{M}_{2 a^{*}}^{\beta s}\left(|f|^{s}\right)(x)\right]^{1 / s} .
\end{aligned}
$$

Combining the estimates of I and J yields

$$
\frac{1}{\gamma\left(B^{\prime}\right)} \int_{B^{\prime}}\left|\mathcal{J}_{3}^{B^{\prime}}(y)-\mathcal{J}_{3}^{B^{\prime}}\left(c_{B^{\prime}}\right)\right| d \gamma(y) \lesssim\|b\|_{*}\left[\mathcal{M}_{2 a^{*}}^{\beta s}\left(|f|^{s}\right)(x)\right]^{1 / s}
$$

Applying (4.7), (4.8) and (4.9), we obtain (4.5), which completes the proof of Lemma 4.2.

Proof of Theorem 1.1 To show (i), we let $b \in \operatorname{BMO}(\gamma)$. For any given $N \in \mathbb{N}$, set $b_{N} \equiv-N \chi_{\{b<-N\}}+b \chi_{\{|b| \leq N\}}+N \chi_{\{b>N\}}$. Then $\left\|b_{N}\right\|_{*} \leq 4\|b\|_{*}$; see [6, pp. 631-632]. Let $\widetilde{C}_{a}$ and $C_{a}$ be respectively as in (4.3) and Lemma 4.2. For all $f \in L_{c}^{\infty}(\gamma)$, combining (4.1), (4.3), (2.2) and Theorem 3.1 yields

$$
\left\|\widetilde{T_{a}^{\beta}\left(b_{N} ; f\right)}\right\|_{L^{q}(\gamma)} \leq\left\|T_{\widetilde{C}_{a}}^{\beta}\left(b_{N} ; f\right)\right\|_{L^{q}(\gamma)} \lesssim N\left\|I_{\widetilde{C}_{a}}^{\beta}(|f|)\right\|_{L^{q}(\gamma)} \lesssim N\|f\|_{L^{p}(\gamma)}<\infty .
$$

This allows us to use (2.7). Thus, by (2.7), Lemma 4.2 together with the fact $1<r, s<p$, the $L^{q / r}(\gamma)$-boundedness of $\mathcal{M}_{1}$, Theorem 3.1, Corollary 3.1 and Lemma 4.1, we obtain

$$
\begin{aligned}
& \left\|T_{a}^{\beta}\left(b_{N} ; f\right)\right\| \|_{L^{q}(\gamma)} \\
& \leq\left\|\widetilde{T_{a}^{\beta\left(b_{N} ; f\right)}}\right\|_{L^{q}(\gamma)} \\
& \lesssim\left\|\widetilde{\left(T_{a}^{\beta\left(b_{N} ; f\right)}\right)^{\sharp}}\right\|_{L^{q}(\gamma)}+\left\|\widetilde{T_{a}^{\beta\left(b_{N} ; f\right)}}\right\|_{L^{1}(\gamma)} \\
& \lesssim\left\|b_{N}\right\|_{*}\left\{\left\|\left[\mathcal{M}_{1}\left(\left[I_{C_{a}}^{\beta}(|f|)\right]^{r}\right)\right]^{1 / r}\right\|_{L^{q}(\gamma)}+\left\|\left[\mathcal{M}_{C_{a}}^{\beta s}\left(|f|^{s}\right)\right]^{1 / s}\right\|_{L^{q}(\gamma)}+\|f\|_{L^{p}(\gamma)}\right\} \\
& \lesssim\|b\|_{*}\|f\|_{L^{p}(\gamma)} .
\end{aligned}
$$

The dominated convergence theorem gives that $b_{N} \rightarrow b$ in $L^{p}(\gamma)$ of every compact set and, in particular, in $L^{p}(d \gamma, \operatorname{supp}(f))$. Hence, $b_{N}|f| \rightarrow b|f|$ in $L^{p}(\gamma)$. From Theorem 3.1, it 
follows easily that both

$$
\int_{\substack{B\left(x, a x_{(x))} \\ b_{N}(x)>b_{N}(y)\right.}} \frac{b_{N}(y)|f(y)|}{\left[e^{\left.-|y|^{2}|x-y|^{n}\right]^{1-\beta}}\right.} d \gamma(y) \rightarrow \int_{\substack{B(x, a m(x)) \\ b(x)>b(y)}} \frac{b(y)|f(y)|}{\left[e^{\left.-|y|^{2}|x-y|^{n}\right]^{1-\beta}}\right.} d \gamma(y)
$$

and

$$
\int_{\substack{B\left(x, a x_{(x))} \\ b_{N}(x) \leq b_{N}(y)\right.}} \frac{b_{N}(y)|f(y)|}{\left[e^{\left.-|y|^{2}|x-y|^{n}\right]^{1-\beta}}\right.} d \gamma(y) \rightarrow \int_{\substack{B(x, a m(x)) \\ b(x) \leq b(y)}} \frac{b(y)|f(y)|}{\left[e^{\left.-|y|^{2}|x-y|^{n}\right]^{1-\beta}}\right.} d \gamma(y)
$$

as $N \rightarrow \infty$ in $L^{q}(\gamma)$. The same is true for (4.10) and (4.11) without $b_{N}$ and $b$ appearing in the integrand functions. These observations together with the Riesz lemma and (4.1) imply that there exists a subsequence $\left\{N_{k}\right\}_{k=1}^{\infty} \subset \mathbb{N}$ such that $T_{a}^{\beta}\left(b_{N_{k}} ; f\right) \rightarrow T_{a}^{\beta}(b ; f)$ as $k \rightarrow \infty$ almost everywhere. Letting $k \rightarrow \infty$ and using Fatou's lemma together with (4.4), we obtain that for all $f \in L_{c}^{\infty}(\gamma)$,

$$
\left\|\widetilde{\left[b, I_{a}^{\beta}\right]}(f)\right\|_{L^{q}(\gamma)} \lesssim\left\|T_{a}^{\beta}(b ; f)\right\|_{L^{q}(\gamma)} \leq \lim _{k \rightarrow \infty}\left\|T_{a}^{\beta}\left(b_{N_{k}} ; f\right)\right\|_{L^{q}(\gamma)} \lesssim\|b\|_{*}\|f\|_{L^{p}(\gamma)} .
$$

Applying the density of $L_{c}^{\infty}(\gamma)$ in the classical Lebesgue space $L^{p}\left(\mathbb{R}^{n}\right)$ and the fact that $\gamma(E) \lesssim|E|$ for all set $E \subset \mathbb{R}^{n}$, one can deduce that $L_{c}^{\infty}(\gamma)$ is dense in $L^{p}(\gamma)$. Moreover, for any $f, g \in L_{c}^{\infty}(\gamma)$, it is easy to deduce that

$$
\left|\widetilde{\left[b, I_{a}^{\beta}\right]}(f+g)\right| \leq\left|\widetilde{\left[b, I_{a}^{\beta}\right]}(f)\right|+\left|\widetilde{\left[b, I_{a}^{\beta}\right]}(g)\right|
$$

and

$$
\left|\widetilde{\left[b, I_{a}^{\beta}\right]}(f)-\widetilde{\left[b, I_{a}^{\beta}\right]}(g)\right| \leq\left|\widetilde{\left[b, I_{a}^{\beta}\right]}(f-g)\right| .
$$

These combined with a standard density argument imply that $\widetilde{\left[b, I_{a}^{\beta}\right]}$ admits a unique bounded extension from $L^{p}(\gamma)$ to $L^{q}(\gamma)$.

Now we show (ii) by borrowing some ideas from [12]. Since $|z|^{n(1-\beta)}$ is infinitely differentiable in any open set away from 0 , we choose $z_{0} \in \mathbb{R}^{n} \backslash\{0\}$ and $\delta>0$ small enough such that the function $|z|^{n(1-\beta)}$ can be expressed as an absolutely convergent Fourier series in the neighborhood $\left\{z \in \mathbb{R}^{n}:\left|z-z_{0}\right|<2 \delta\right\}$, that is,

$$
|z|^{n(1-\beta)}=\sum_{m \in \mathbb{Z}^{n}} a_{m} e^{i m \cdot z}
$$

see, for example, [12, p. 266].

Set $z_{1} \equiv \delta^{-1} z_{0}$. Choose $\widetilde{a} \in(0, \infty)$ such that $a=\widetilde{a}(\widetilde{a}+1)\left(2+\left|z_{1}\right|\right)$. For any $B \in \mathcal{B}_{\widetilde{a}}$, we denote by $B^{\prime}$ the ball centered at $c_{B}-r_{B} z_{1}$ with radius $r_{B}$. Notice that

$$
B^{\prime} \subset B\left(c_{B},\left(1+\left|z_{1}\right|\right) r_{B}\right) \subset B\left(c_{B^{\prime}},\left(1+2\left|z_{1}\right|\right) r_{B}\right) .
$$


This combined with (2.1) implies that $\gamma(B) \sim \gamma\left(B^{\prime}\right)$ with the equivalent constants depending on $z_{1}$. Moreover, for any $x \in B$ and $y \in B^{\prime}$, we have

$$
\left|\frac{\delta(x-y)}{r_{B}}-z_{0}\right| \leq\left|\frac{\delta\left(x-c_{B}\right)}{r_{B}}\right|+\left|\frac{\delta\left(c_{B}-y\right)}{r_{B}}-z_{0}\right|<2 \delta
$$

Set $s(x) \equiv \operatorname{sgn}\left[b(x)-b_{B^{\prime}}\right]$. Notice that for any $y \in B^{\prime}$ and $x \in B$, by (2.4), we obtain

$$
\begin{aligned}
|y-x| & \leq\left|y-c_{B^{\prime}}\right|+\left|c_{B^{\prime}}-c_{B}\right|+\left|c_{B}-x\right| \\
& <\left(2+\left|z_{1}\right|\right) r_{B} \leq\left(2+\left|z_{1}\right|\right) \widetilde{a} m\left(c_{B}\right) \leq \widetilde{a}(\widetilde{a}+1)\left(2+\left|z_{1}\right|\right) m(x) ;
\end{aligned}
$$

thus, $B^{\prime} \subset B(x, a m(x))$ for all $x \in B$. Then we have

$$
\begin{aligned}
\int_{B}\left|b(x)-b_{B^{\prime}}\right| d \gamma(x) \\
=\frac{\left(r_{B}\right)^{n(1-\beta)}}{\gamma\left(B^{\prime}\right)} \int_{B} \int_{B^{\prime}} \frac{b(x)-b(y)}{[\delta|x-y|]^{n(1-\beta)}}\left|\frac{\delta(x-y)}{r_{B}}\right|^{n(1-\beta)} s(x) d \gamma(y) d \gamma(x) \\
=\frac{\left(\delta^{-1} r_{B}\right)^{n(1-\beta)}}{\gamma\left(B^{\prime}\right)} \sum_{m \in \mathbb{Z}^{n}} a_{m} \int_{\mathbb{R}^{n}} \int_{B(x, a m(x))} \frac{b(x)-b(y)}{\left[e^{\left.-|x|^{2}|x-y|^{n}\right]^{1-\beta}} e^{i m \cdot \frac{\delta(x-y)}{r_{B}}} e^{-|x|^{2}(1-\beta)}\right.} \\
\quad \times s(x) \chi_{B}(x) \chi_{B^{\prime}}(y) d \gamma(y) d \gamma(x) .
\end{aligned}
$$

If we set $f_{m}(y) \equiv e^{-i m \cdot \frac{\delta y}{r_{B}}} \chi_{B^{\prime}}(y)$ for all $y \in \mathbb{R}^{n}$ and $g_{m}(x) \equiv e^{i m \cdot \frac{\delta x}{r_{B}}} s(x) \chi_{B}(x)$ for all $x \in \mathbb{R}^{n}$, then the last formula above equals to a constant multiple of

$$
\frac{\left(\delta^{-1} r_{B}\right)^{n(1-\beta)}}{\gamma\left(B^{\prime}\right)} \sum_{m \in \mathbb{Z}^{n}} a_{m} \int_{\mathbb{R}^{n}}\left[b, \widetilde{I}_{a}^{\beta}\right]\left(f_{m}\right)(x) e^{-|x|^{2}(1-\beta)} g_{m}(x) d \gamma(x) .
$$

Notice that $B^{\prime} \subset B\left(c_{B},\left(1+\left|z_{1}\right|\right) r_{B}\right)$ and (2.4) imply that $e^{-\left|c_{B}\right|^{2}} \sim e^{-\left|c_{B^{\prime}}\right|^{2}}$. By this, supp $g_{m} \subset B$ and the fact $e^{-|x|^{2}} \sim e^{-\left|c_{B}\right|^{2}}$ for all $x \in B$, we obtain that $e^{-|x|^{2}} \sim e^{-\left|c_{B^{\prime}}\right|^{2}}$ for all $x \in B$. From this and (2.2) together with Hölder's inequality, we deduce that

$$
\begin{aligned}
\int_{B}\left|b(x)-b_{B^{\prime}}\right| d \gamma(x) & \lesssim \frac{\left(\delta^{-1} r_{B}\right)^{n(1-\beta)} e^{-\left|c_{B^{\prime}}\right|^{2}(1-\beta)}}{\gamma\left(B^{\prime}\right)} \sum_{m \in \mathbb{Z}^{n}}\left|a_{m}\right| \int_{B}\left|\left[b, \widetilde{I}_{a}^{\beta}\right]\left(f_{m}\right)(x)\right| d \gamma(x) \\
& \lesssim\left[\gamma\left(B^{\prime}\right)\right]^{-\beta}[\gamma(B)]^{1 / q^{\prime}} \sum_{m \in \mathbb{Z}^{n}}\left|a_{m}\right|\left\|\left[b, \widetilde{I}_{a}^{\beta}\right]\left(f_{m}\right)\right\|_{L^{q}(\gamma)} \\
& \lesssim\left\|\left[b, \widetilde{I}_{a}^{\beta}\right]\right\|_{L^{p}(\gamma) \rightarrow L^{q}(\gamma)} \gamma(B) .
\end{aligned}
$$

It follows that $\frac{1}{\gamma(B)} \int_{B}\left|b(x)-b_{B}\right| d \gamma(x) \lesssim\left\|\left[b, \widetilde{I}_{a}^{\beta}\right]\right\|_{L^{p}(\gamma) \rightarrow L^{q}(\gamma)}$. Taking the supermum over all balls $B \in \mathcal{B}_{a}$ yields that $\|b\|_{*} \lesssim\left\|\left[b, \widetilde{I}_{a}^{\beta}\right]\right\|_{L^{p}(\gamma) \rightarrow L^{q}(\gamma)}$. Hence, we complete the proof of Theorem 1.1. 
Proof of Theorem 1.2 To show (i), by (2.2) and (2.3) together with an argument similar to that used in the proof of Corollary 3.1, we obtain

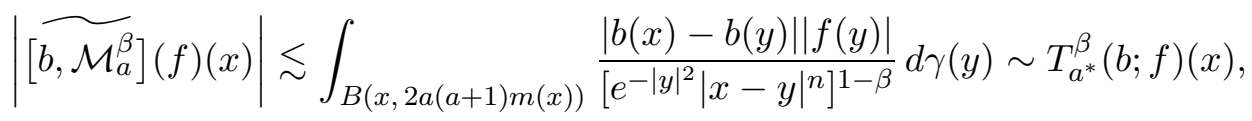

where $a^{*} \equiv 2 a(a+1)$. Then following the argument used in the proof of Theorem 1.1 (i) yields that $\left[b, \mathcal{M}_{a}^{\beta}\right]$ admits a unique bounded extension from $L^{p}(\gamma)$ to $L^{q}(\gamma)$ with norm at most a constant multiple of $\|b\|_{*}$.

Next we show (ii). Using Hölder's inequality and the boundedness of $\left[\widetilde{b, \mathcal{M}_{a}^{\beta}}\right]$, we obtain that for all $B \in \mathcal{B}_{a}$,

$$
\begin{aligned}
\frac{1}{\gamma(B)} \int_{B}\left|b(y)-b_{B}\right| d \gamma(y) & \leq \frac{1}{[\gamma(B)]^{1+\beta}} \int_{B} \frac{1}{[\gamma(B)]^{1-\beta}} \int_{B}|b(y)-b(x)| \chi_{B}(x) d \gamma(x) d \gamma(y) \\
& \leq \frac{1}{[\gamma(B)]^{1+\beta}} \int_{B}\left[\frac{\left[b \mathcal{M}_{a}^{\beta}\right.}{[}\right]\left(\chi_{B}\right)(y) d \gamma(y) \\
& \left.\leq[\gamma(B)]^{1 / q^{\prime}-1-\beta} \| \widetilde{\left[b, \mathcal{M}_{a}^{\beta}\right.}\right]\left(\chi_{B}\right)\left\|_{L^{q}(\gamma)} \leq\right\| \widetilde{\left[b, \mathcal{M}_{a}^{\beta}\right]} \|_{L^{p}(\gamma) \rightarrow L^{q}(\gamma)},
\end{aligned}
$$

which together with the fact $b \in L^{1}(\gamma)$ further implies that $b \in \operatorname{BMO}(\gamma)$. Thus, we complete the proof of Theorem 1.2.

Proof of Theorem 1.3 First we prove (i). Observe that for all $x \in \mathbb{R}^{n}$,

$$
\left.\left[b, \mathcal{M}_{a}^{\beta}\right](f)(x)=b(x) \mathcal{M}_{a}^{\beta}(f)(x)-\mathcal{M}_{a}^{\beta}(b f)(x) \leq \widetilde{\left[b, \mathcal{M}_{a}^{\beta}\right.}\right](f)(x) .
$$

Moreover, if we further assume that $b$ is a nonnegative function, then for all $x \in \mathbb{R}^{n}$,

$$
\left.\left|\left[b, \mathcal{M}_{a}^{\beta}\right](f)(x)\right| \leq \widetilde{\left[b, \mathcal{M}_{a}^{\beta}\right.}\right](f)(x) .
$$

For all $x \in \mathbb{R}^{n}$, since

$$
\begin{aligned}
-\left[b, \mathcal{M}_{a}^{\beta}\right](f)(x) & \leq \mathcal{M}_{a}^{\beta}\left(b^{+} f\right)(x)+\mathcal{M}_{a}^{\beta}\left(b^{-} f\right)(x)-b^{+}(x) \mathcal{M}_{a}^{\beta}(f)(x)+b^{-}(x) \mathcal{M}_{a}^{\beta}(f)(x) \\
& \leq\left|\left[b^{+}, \mathcal{M}_{a}^{\beta}\right](f)(x)\right|+\mathcal{M}_{a}^{\beta}\left(b^{-} f\right)(x)+b^{-}(x) \mathcal{M}_{a}^{\beta}(f)(x)
\end{aligned}
$$

we then apply $(4.13)$ and the fact $\left.\left[\widetilde{b^{+}, \mathcal{M}_{a}^{\beta}}\right](f)(x) \leq \widetilde{b, \mathcal{M}_{a}^{\beta}}\right](f)(x)$ to obtain

$$
\left.-\left[b, \mathcal{M}_{a}^{\beta}\right](f)(x) \leq \widetilde{\left[b, \mathcal{M}_{a}^{\beta}\right.}\right](f)(x)+\mathcal{M}_{a}^{\beta}\left(b^{-} f\right)(x)+b^{-}(x) \mathcal{M}_{a}^{\beta}(f)(x),
$$

which together with (4.12) yields

$$
\left.\left|\left[b, \mathcal{M}_{a}^{\beta}\right](f)(x)\right| \leq \widetilde{\left[b, \mathcal{M}_{a}^{\beta}\right.}\right](f)(x)+\mathcal{M}_{a}^{\beta}\left(b^{-} f\right)(x)+b^{-}(x) \mathcal{M}_{a}^{\beta}(f)(x) .
$$

Then applying Theorem 1.2 (i) and Corollary 3.1 together with the assumption $b^{-} \in L^{\infty}(\gamma)$ yields Theorem 1.3 (i). 
To prove (ii), we first show $b \in \operatorname{BMO}(\gamma)$. To this end, fix $B \in \mathcal{B}_{a}$. Then set

$$
E \equiv\left\{x \in B: b(x) \leq b_{B}\right\}
$$

Recall that $b_{B}$ is the integral average over the ball $B$. It is not difficult to see that

$$
\int_{E}\left|b(x)-b_{B}\right| d \gamma(x)=\int_{B \backslash E}\left|b(x)-b_{B}\right| d \gamma(x) .
$$

Thus,

$$
\begin{aligned}
\frac{1}{\gamma(B)} \int_{B}\left|b(x)-b_{B}\right| d \gamma(x) & =\frac{2}{\gamma(B)} \int_{E}\left[b_{B}-b(x)\right] d \gamma(x) \\
& =\frac{2}{[\gamma(B)]^{2}} \int_{E} \int_{B}[b(y)-b(x)] d \gamma(y) d \gamma(x)
\end{aligned}
$$

For all $x \in B$ and $B \in \mathcal{B}_{a}$, we have

$$
\begin{aligned}
& \sup _{\widetilde{B} \in \mathcal{B}_{a}(x)} \frac{\gamma(B \cap \widetilde{B})}{[\gamma(\widetilde{B})]^{1-\beta}[\gamma(B)]^{\beta}} \\
& \quad=\max \left\{\sup _{\substack{\widetilde{B} \in \mathcal{B}_{a}(x) \\
\gamma(B) \geq \gamma(\widetilde{B})}} \frac{\gamma(B \cap \widetilde{B})}{\gamma(\widetilde{B})}\left(\frac{\gamma(\widetilde{B})}{\gamma(B)}\right)^{\beta}, \sup _{\substack{\widetilde{B} \in \mathcal{B}_{a}(x) \\
\gamma(B)<\gamma(\widetilde{B})}} \frac{\gamma(B \cap \widetilde{B})}{\gamma(B)}\left(\frac{\gamma(B)}{\gamma(\widetilde{B})}\right)^{1-\beta}\right\} \leq 1,
\end{aligned}
$$

which further implies that

$$
\sup _{\widetilde{B} \in \mathcal{B}_{a}(x)} \frac{\gamma(B \cap \widetilde{B})}{[\gamma(\widetilde{B})]^{1-\beta}[\gamma(B)]^{\beta}}=1 .
$$

Equivalently speaking, for all $B \in \mathcal{B}_{a}$ and $x \in B$,

$$
\mathcal{M}_{a}^{\beta}\left(\chi_{B}\right)(x)=[\gamma(B)]^{\beta} .
$$

This combined with (1.5) and (1.6) yields that for all $x \in \mathbb{R}^{n}$,

$$
\begin{aligned}
\left|\left[b, \mathcal{M}_{a}^{\beta}\right]\left(\chi_{B}\right)(x)\right| & \geq \sup _{\widetilde{B} \in \mathcal{B}_{a}(x)} \frac{1}{[\gamma(\widetilde{B})]^{1-\beta}} \int_{\widetilde{B}} b(y) \chi_{B}(y) d \gamma(y)-b(x) \mathcal{M}_{a}^{\beta}\left(\chi_{B}\right)(x) \\
& \geq \frac{1}{[\gamma(B)]^{1-\beta}} \int_{B}[b(y)-b(x)] d \gamma(y) .
\end{aligned}
$$

Inserting this into (4.14) yields

$$
\begin{aligned}
\frac{1}{\gamma(B)} \int_{B}\left|b(x)-b_{B}\right| d \gamma(x) & \leq \frac{2}{[\gamma(B)]^{1+\beta}} \int_{E}\left|\left[b, \mathcal{M}_{a}^{\beta}\right]\left(\chi_{B}\right)(x)\right| d \gamma(x) \\
& \leq \frac{2}{[\gamma(B)]^{1+\beta}} \int_{B}\left|\left[b, \mathcal{M}_{a}^{\beta}\right]\left(\chi_{B}\right)(x)\right| d \gamma(x)
\end{aligned}
$$


Then using Hölder's inequality and the boundedness of $\left[b, \mathcal{M}_{a}^{\beta}\right]$ from $L^{p}(\gamma)$ to $L^{q}(\gamma)$, we further obtain that for all $B \in \mathcal{B}_{a}$,

$$
\frac{1}{\gamma(B)} \int_{B}\left|b(x)-b_{B}\right| d \gamma(x) \leq \frac{2[\gamma(B)]^{1 / q^{\prime}}}{\gamma(B)^{1+\beta}}\left\|\left[b, \mathcal{M}_{a}^{\beta}\right]\left(\chi_{B}\right)\right\|_{L^{q}(\gamma)} \leq 2\left\|\left[b, \mathcal{M}_{a}^{\beta}\right]\right\|_{L^{p}(\gamma) \rightarrow L^{q}(\gamma)}
$$

Taking the supremum over all balls $B \in \mathcal{B}_{a}$ yields $\|b\|_{*}^{\mathcal{B}_{a}, 1} \lesssim\left\|\left[b, \mathcal{M}_{a}^{\beta}\right]\right\|_{L^{p}(\gamma) \rightarrow L^{q}(\gamma)}$. This and the hypothesis $b \in L^{1}(\gamma)$ together with (2.8) and (1.1) imply that $b \in \operatorname{BMO}(\gamma)$.

We still need to prove that $b^{-} \in L^{\infty}(\gamma)$. The differentiation theorem for the integral implies that for almost all $x \in \mathbb{R}^{n}$,

$$
b^{+}(x)=\lim _{B \in \mathcal{B}_{a}(x), r_{B} \rightarrow 0} \frac{1}{\gamma(B)} \int_{B} b^{+}(z) d \gamma(z) .
$$

Fix $x$ satisfying (4.16). Then for any given $\epsilon>0$, there exists $B_{0} \in \mathcal{B}_{a}(x)$ such that for all $B \in \mathcal{B}_{a}(x)$ and $B \subset B_{0}$, we have

$$
\left|b^{+}(x)-\frac{1}{\gamma(B)} \int_{B} b^{+}(z) d \gamma(z)\right|<\epsilon,
$$

and hence,

$$
b^{+}(x) \leq \frac{1}{\gamma(B)} \int_{B} b^{+}(z) d \gamma(z)+\epsilon .
$$

It follows that for $x$ satisfying (4.16),

$$
\begin{aligned}
b^{-}(x) & \leq \frac{1}{\gamma(B)} \int_{B} b^{+}(z) d \gamma(z)+\epsilon-b^{+}(x)+b^{-}(x) \\
& =\frac{1}{\gamma(B)} \int_{B} b^{+}(z) d \gamma(z)+\epsilon-b(x) \leq \frac{1}{\gamma(B)} \int_{B}|b(z)| d \gamma(z)-b(x)+\epsilon .
\end{aligned}
$$

For the above $B$ satisfying $B \in \mathcal{B}_{a}(x)$ and $B \subset B_{0}$, we use (4.15) to obtain that

$$
\begin{aligned}
\frac{1}{\gamma(B)} \int_{B}|b(z)| d \gamma(z)-b(x) & =\frac{1}{\gamma(B)} \int_{B}|b(z)| d \gamma(z)-b(x) \mathcal{M}_{a}^{\beta}\left(\chi_{B}\right)(x)[\gamma(B)]^{-\beta} \\
& \leq[\gamma(B)]^{-\beta}\left|\left[b, \mathcal{M}_{a}^{\beta}\right]\left(\chi_{B}\right)(x)\right| .
\end{aligned}
$$

Inserting this into (4.17) yields

$$
b^{-}(x) \leq[\gamma(B)]^{-\beta}\left|\left[b, \mathcal{M}_{a}^{\beta}\right]\left(\chi_{B}\right)(x)\right|+\epsilon .
$$

We take the integration average over $B$ on both sides of (4.18), then use Hölder's inequality and the boundedness of $\left[b, \mathcal{M}_{a}^{\beta}\right]$, and finally obtain

$$
\begin{aligned}
\frac{1}{\gamma(B)} \int_{B} b^{-}(x) d \gamma(x) & \leq \frac{1}{\gamma(B)^{1+\beta}} \int_{B}\left|\left[b, \mathcal{M}_{a}^{\beta}\right]\left(\chi_{B}\right)(x)\right| d \gamma(x)+\epsilon \\
& \leq[\gamma(B)]^{1 / q^{\prime}-1-\beta}\left\|\left[b, \mathcal{M}_{a}^{\beta}\right]\left(\chi_{B}\right)\right\|_{L^{q}(\gamma)}+\epsilon \leq\left\|\left[b, \mathcal{M}_{a}^{\beta}\right]\right\|_{L^{p}(\gamma) \rightarrow L^{q}(\gamma)}+\epsilon .
\end{aligned}
$$


This combined with the differentiation theorem for the integral implies that for the points $y \in B_{0}$ such that (4.16) holds with $b^{+}$replaced by $b^{-}$,

$$
b^{-}(y) \leq\left\|\left[b, \mathcal{M}_{a}^{\beta}\right]\right\|_{L^{p}(\gamma) \rightarrow L^{q}(\gamma)}+\epsilon .
$$

So for the point $x$ satisfies (4.16) and also satisfies (4.16) with $b^{+}$replaced by $b^{-}$, we have

$$
b^{-}(x) \leq\left\|\left[b, \mathcal{M}_{a}^{\beta}\right]\right\|_{L^{p}(\gamma) \rightarrow L^{q}(\gamma)}+\epsilon .
$$

Letting $\epsilon \rightarrow 0$ yields

$$
\left\|b^{-}\right\|_{L^{\infty}(\gamma)} \leq\left\|\left[b, \mathcal{M}_{a}^{\beta}\right]\right\|_{L^{p}(\gamma) \rightarrow L^{q}(\gamma)} .
$$

Thus, we obtain the desired results of (ii). This finishes the proof of Theorem 1.3.

\section{References}

[1] D. R. Adams, A note on Riesz potentials, Duke Math. J. 42(1975), 765-778.

[2] J. Bastero, M. Milman and F. J. Ruiz, Commutators for the maximal and sharp functions, Proc. Amer. Math. Soc. 128(2000), 3329-3334.

[3] S. Chanillo, A note on commutators, Indiana Univ. Math. J. 31(1982), 7-16.

[4] R. R. Coifman, R. Rochberg and G. Weiss, Factorization theorems for Hardy spaces in several variables, Ann. of Math. 103(1976), 611-635.

[5] R. R. Coifman and G. Weiss, Analyse Harmonique Non-commutative sur Certains Espaces Homogènes, Lecture Notes in Math. 242, Springer, Berlin, 1971.

[6] R. R. Coifman and G. Weiss, Extensions of Hardy spaces and their use in analysis, Bull. Amer. Math. Soc. 83(1977), 569-645.

[7] J. García-Cuerva, G. Mauceri, P. Sjögren and J. L. Torrea, Higher-order Riesz operators for the Ornstein-Uhlenbeck semigroup, Potential Anal. 10(1999), 379-407.

[8] L. Grafakos, Modern Fourier Analysis, Second Edition, Graduate Texts in Math., No. 250, Springer, New York, 2008.

[9] C. E. Gutiérrez, On the Riesz transforms for Gaussian measures, J. Funct. Anal. 120(1994), 107-134.

[10] P. Hajłasz and P. Koskela, Sobolev met Poincaré, Mem. Amer. Math. Soc. 145(2000), 1-101.

[11] L. Hedberg, On certain convolution inequalities, Proc. Amer. Math. Soc. 36(1972), 505-510.

[12] S. Janson, Mean oscillation and commutators of singular integral operators, Ark. Mat. 16(1978), 263-270.

[13] F. John and L. Nirenberg, On functions of bounded mean oscillation, Comm. Pure Appl. Math. 14(1961), 415-426.

[14] L. Liu and D. Yang, BLO spaces associated with the Ornstein-Uhlenbeck operator, Bull. Sci. Math. 132(2008), 633-649.

[15] S. Lu, Y. Ding and D. Yan, Singular Integrals and Related Topics, World Scientific Publishing Co. Pte. Ltd., Hackensack, NJ, 2007. 
[16] G. Mauceri and S. Meda, BMO and $H^{1}$ for the Ornstein-Uhlenbeck operator, J. Funct. Anal. 252(2007), 278-313.

[17] S. Pérez, The local part and the strong type for operators related to the Gaussian measure, J. Geom. Anal. 11(2001), 491-507.

[18] G. Pisier, Riesz transforms: a simpler analytic proof of P.-A. Meyer's inequality, Lecture Notes in Math., 1321(1988), 485-501.

[19] P. Sjögren, Operators associated with the Hermite operator-A survey, J. Fourier Anal. Appl. 3(1997), 813-823.

[20] E. M. Stein, Singular Integrals and Differentiability Properties of Functions, Princeton University Press, Princeton, N. J., 1970.

[21] E. M. Stein, Harmonic Analysis: Real-Variable Methods, Orthogonality, and Oscillatory Integrals, Princeton University Press, Princeton, N. J., 1993.

[22] E. M. Stein and G. Weiss, On the theory of harmonic functions of several variables, I: The theory of $H^{p}$ spaces, Acta Math. 103(1960), 25-62.

[23] A. Torchinsky, Real-Variable Methods in Harmonic Analysis, Academic Press, Inc., Orlando, F. L., 1986.

Liguang LiU \& Dachun YAng (Corresponding author)

School of Mathematical Sciences, Beijing Normal University, Laboratory of Mathematics and Complex Systems, Ministry of Education, Beijing 100875, People's Republic of China

E-mails: liuliguang@mail.bnu.edu.cn \& dcyang@bnu.edu.cn 